\title{
Existence and multiplicity of solutions for the fractional $p$-Laplacian Choquard logarithmic equation involving a nonlinearity with exponential critical and subcritical growth *
}

\author{
Eduardo de S. Böer tand Olímpio H. Miyagaki ${ }^{\S}$ \\ Department of Mathematics, Federal University of São Carlos, \\ 13565-905 São Carlos, SP - Brazil
}

December 24, 2020

\begin{abstract}
In the present work we obtain the existence and multiplicity of nontrivial solutions for the Choquard logarithmic equation $(-\Delta)_{p}^{s} u+|u|^{p-2} u+\left(\ln |\cdot| *|u|^{p}\right)|u|^{p-2} u=f(u)$ in $\mathbb{R}^{N}$ , where $N=s p, s \in(0,1), p>2, a>0, \lambda>0$ and $f: \mathbb{R} \rightarrow \mathbb{R}$ a continuous nonlinearity with exponential critical and subcritical growth. We guarantee the existence of a nontrivial solution at the mountain pass level and a nontrivial ground state solution under critical and subcritical growth. Morever, when $f$ has subcritical growth we prove the existence of infinitely many solutions, via genus theory.
\end{abstract}

Mathematics Subject Classification: 35J60, 35J15, 35Q55, 335B25.

Key words. Choquard logarithmic equations, exponential growth, variational techniques, ground state solution.

\section{Introduction}

In the present paper we study existence and multiplicity results for the Choquard logarithmic equation

$$
(-\Delta)_{p}^{s} u+|u|^{p-2} u+\left(\ln |\cdot| *|u|^{p}\right)|u|^{p-2} u=f(u) \text { in } \mathbb{R}^{N},
$$

where $N=s p, s \in(0,1), p>2, a=1, \lambda=1$ and $f: \mathbb{R} \rightarrow \mathbb{R}$ is continuous, with primitive $F(t)=\int_{0}^{t} f(\tau) d \tau$. Precisely, when dealing with critical exponential growth, we will

*The first author was supported by Coordination of Superior Level Staff Improvement-(CAPES) -Finance Code 001 and São Paulo Research Foundation- (FAPESP), grant $\sharp 2019 / 22531$-4, while the second author was supported by National Council for Scientific and Technological Development -(CNPq), grant $\sharp$ 307061/2018-3 and FAPESP grant $\sharp 2019 / 24901-3$.

† Corresponding author

†E-mail address: eduardoboer04@gmail.com Tel. +55.51.993673377

${ }^{\S}$ E-mail address: ohmiyagaki@gmail.com, Tel.: +55.16.33519178 (UFSCar). 
guarantee the existence of a solution at the mountain pass level and a ground state solution, in the sense that it will be the least energy level. On the other hand, considering $f$ with subcritical exponential growth, we are able to prove that equation (1.1) has infinitely many solutions, via genus theory. Such nonlinearity behaviour is motivated by the Moser-Trudinger Lemma 2.2.

First of all, to make the notation concise, we set, for $\alpha>0$ and $t \in \mathbb{R}$,

$$
R(\alpha, t)=\exp \left(\alpha|t|^{\frac{N}{N-s}}\right)-S_{k_{p}-2}(\alpha, t)=\sum_{k_{p}-1}^{+\infty} \frac{\alpha^{k}}{k !}|s|^{\frac{N}{N-s} k}
$$

where $S_{k_{p}-2}(\alpha, t)=\sum_{k=0}^{k_{p}-2} \frac{\alpha^{k}}{k !}|t|^{\frac{N}{N-s}} k$ and $k_{p}=\min \{k \in \mathbb{N} ; k \geq p\}$.

Then, we recall that a function $h$ has subcritical exponential growth at $+\infty$, if

$$
\lim _{t \rightarrow+\infty} \frac{h(t)}{R(\alpha, t)}=0, \text { for all } \alpha>0,
$$

and we say that $h$ has critical exponential growth at $+\infty$, if there exists $\alpha_{0}>0$ such that

$$
\lim _{t \rightarrow+\infty} \frac{h(t)}{R(\alpha, t)}=\left\{\begin{array}{l}
0, \quad \forall \alpha>\alpha_{0} \\
+\infty, \quad \forall \alpha<\alpha_{0}
\end{array}\right.
$$

In the following we present some necessary conditions to obtain our main results. This kind of hypothesis are usual in works with Moser-Trudinger inequality, such as $[5,15,16]$. We assume that $f$ satisfies

$\left(f_{1}\right) \quad f \in C(\mathbb{R}, \mathbb{R}), f(0)=0$, has critical exponential growth and $F(t) \geq 0$, for all $t \in \mathbb{R}$.

$$
\lim _{|t| \rightarrow 0} \frac{f(t)}{|t|^{p-2} t}=0
$$

From $\left(f_{1}\right)$ and $\left(f_{2}\right)$, given $\varepsilon>0$ and $\alpha>\alpha_{0}$, fixed, there exists a constant $b_{1}>0$ such that

$$
|f(t)| \leq \varepsilon|t|^{p-1}+b_{1} R(\alpha, t), \quad \forall t \in \mathbb{R}
$$

As a consequence,

$$
|F(t)| \leq \frac{\varepsilon}{p}|t|^{p}+b_{1}|t| R(\alpha, t), \quad \forall t \in \mathbb{R} .
$$

Our strategy to prove Theorem 1.1 will consist in finding a Cerami sequence for the mountain pass level. In order to verify that such sequence is bounded in $W^{s, p}\left(\mathbb{R}^{N}\right)$, we will need the following condition

$$
\text { there exists } \theta>2 p \text { such that } f(t) t \geq \theta F(t)>0 \text {, for all } t>0 \text {. }
$$

Moreover, as we will be working with an exponential term, to guarantee that the mentioned Cerami sequence and the minimizing sequence for the ground state satisfy the exponential esti- 
mates, we rely in the next condition.

$\left(f_{4}\right)$ there exist $q>2 p$ and $C_{q}>\frac{[2(q-p)]^{\frac{q-p}{p}}}{q^{\frac{q}{p}}} \frac{S_{q}^{q}}{\rho_{0}^{q-p}}$ such that $F(t) \geq C_{q}|t|^{q}$, for all $t \in \mathbb{R}$, for $S_{q}, \rho_{0}>0$ to be defined in Lemma 3.7.

Therefore, we are able to state our first main result.

Theorem 1.1. Assume $\left(f_{1}\right)-\left(f_{4}\right), q>2 p$ and $C_{q}>0$ sufficiently large. Then,

(i) Problem (1.1) has a nontrivial solution $u \in X$ such that

$$
I(u)=c_{m p}=\inf _{\gamma \in \Gamma} \max _{t \in[0,1]} I(\gamma(t)),
$$

where $\Gamma=\{\gamma \in C([0,1], X) ; \gamma(0), I(\gamma(1))<0\}$.

(ii) Problem (1.1) has a nontrivial ground state solution $u \in X$, that is, u satisfies

$$
I(u)=c_{g}=\inf \{I(v) ; v \in X \text { is a solution of }(1.1)\} .
$$

For the second main result, we are concerned with multiplicity of solutions. However, to obtain this we need to exchange the condition $\left(f_{1}\right)$ by the condition below.

$\left(f_{1}^{\prime}\right) f \in C(\mathbb{R}, \mathbb{R}), f$ is odd, has subcritical exponential growth and $F(t) \geq 0$, for all $t \in \mathbb{R}$.

Also, we need to add a condition that gives us the desired geometry for the associated functional. That is,

$$
\text { the function } t \mapsto \frac{f(t)}{t^{2 p-1}} \text { is increasing in }(0,+\infty) \text {. }
$$

From this condition, since $f$ is odd, it follows that $\frac{f(t)}{t^{2 p-1}}$ is decreasing in $(-\infty, 0)$.

Moreover, since we can control the exponent using $\alpha>0$, we can change condition $\left(f_{4}\right)$ by a more general condition, that is

$$
\text { there exists } q>2 p \text { and } M_{1}>0 \text { such that } F(t) \geq M_{1}|t|^{q}, \forall t \in \mathbb{R} \text {, }
$$

Theorem 1.2. Suppose $\left(f_{1}^{\prime}\right),\left(f_{2}\right),\left(f_{3}\right),\left(f_{4}^{\prime}\right),\left(f_{5}\right)$. Then, problem (1.1) has infinitely many solutions.

To conclude this introduction, we make a quick overview of the state of art. In the first half of this overview, we will be concerned with fractional $p$-Laplacian equations and problems that deal with exponential nonlinearities. We recall that problems with nonlocal operators arise in many areas, such as optimization, finance, phase transitions, stratified materials, anomalous diffusion, crystal dislocation, soft thin films, semipermeable membranes, flame propagation, conservation laws and water waves. See e.g. [13], where the authors provide an extensive list with references for the mentioned applications. For fractional problems of the form

$$
(-\Delta)_{p}^{s} u+V(x)|u|^{p-2} u=f(x, u)+\varepsilon h(x) \text { in } \mathbb{R}^{N}
$$


we mention [11,25,37]. In the [37], the authors consider problem (1.4) in the case $N=s p$, $s \in(0,1), V(x)$ having a positive lower bound and being coercive or satisfying $\frac{1}{V(x)} \in L^{1}\left(\mathbb{R}^{N}\right)$, $f(x, t)$ behaving like $e^{\alpha|t|^{\frac{N}{N-s}}}$ at infinity, $h \in\left(W^{s, p}\left(\mathbb{R}^{N}\right)\right)^{*}$ and $\varepsilon>0$. Under suitable conditions over $V, f, h$, the authors guarantee the existence of weak solutions for (1.4). In [25], they consider $V \in C\left(\mathbb{R}^{N}\right)$ having a positive lower bound, $p \geq 2, s \in(0,1), N \geq 2, h \equiv 0$ and $f p$-superlinear. Using Mountain Pass Theorem for Cerami condition, the authors prove the existence of a nontrivial radially symmetric solution. Finally, in [11], the authors consider $V \in L^{\infty}\left(\mathbb{R}^{N}\right)$ possibly indefinite, a concave and convex nonlinearity $f(x, u)=w_{1}(x)|u|^{q-2} u-$ $w_{2}(x)|u|^{r-2} u$, for $0<s<1<q<p<r$, and $h \equiv 0$. They begin showing that $(-\Delta)_{p}^{s} u=$ $\lambda V(x)|u|^{p-2} u$ posses a infinite sequence of eigenvalues and that the first one is simple. Then, using this fact, the obtain the existence of infinitely many solutions for (1.4). See also [30].

We also refer the reader for the works [3,16,21,22], in which the authors dealt with fractional Laplacian operator, and the works [8,24,31], for general problems with Moser-Trudinger type behaviour.

In the second half, we take a look into works that deal with Choquard logarithmic equations. We can cite the recent works of $[2,9,10,17,20,35]$, where the authors study the following class of equation

$$
-\Delta u+V(x) u+\gamma\left(\ln |\cdot| *|u|^{2}\right) u=f(u), \quad \text { in } \mathbb{R}^{N},
$$

under distinct conditions. We briefly discuss some of these works and left others to the reader. In [10], the authors have proved the existence of infinitely many geometrically distinct solutions and a ground state solution, considering $N=2, V: \mathbb{R}^{N} \rightarrow(0, \infty)$ continuous and $\mathbb{Z}^{2}$-periodic, $\gamma>0, b \geq 0, f(u)=b|u|^{p-2} u$ and $p \geq 4$. Here because of the periodic setting, the global Palais-Smale condition can fail, since the corresponding functional become invariant under $\mathbb{Z}^{2}$-translations. Then, intending to fill the gap, in [17] the authors proved the existence of a mountain pass solution and a ground state solution for the equation (1.5) in the case $N=2$, $V(x) \equiv a>0, \lambda>0, f(u)=b|u|^{p-2} u$ and $2<p<4$. Also, they verified that, if $p \geq 3$, both levels are equal and provided a characterization for them. In [9], the authors dealt with the existence of stationary waves with prescribed norm considering $\lambda \in \mathbb{R}$. Finally, in [2], the authors proved the existence of a ground state solution for equation (1.5), with a nonlinearity of Moser-Trudinger type. We also refer to $[6,28]$ for Choquard equations.

We also call attention to [5], where the authors consider the following equation

$$
(-\Delta)^{\frac{1}{2}} u+u+\left(\ln |\cdot| *|u|^{2}\right) u=f(u) \text { in } \mathbb{R}
$$

with $f \in C(\mathbb{R}, \mathbb{R})$ a nonlinearity of Moser-Trudinger type. In this work, they prove that (1.6) has a mountain pass solution and a ground state solution, when $f$ has exponential critical behaviour. On the other, consider $f$ odd and with subcritical exponential growth, the authors obtained infinitely many solutions for (1.6). How to obtain multiplicity results for (1.6) in the critical case remains an open problem.

The present work aims to expand and complement those results already found in the literature, considering a Choquard logarithmic equation for the fractional $p$-Laplacian operator with a nonlinearity under exponential growth.

Throughout the paper, we will use the following notations: $L^{\omega}\left(\mathbb{R}^{N}\right)$ denotes the usual 
Lebesgue space with norm $\|\cdot\|_{\omega} ; X^{\prime}$ denotes the dual space of $X ; B_{r}(x)$ is the ball in $\mathbb{R}$ centred in $x$ with radius $r>0$ and simply $B_{r}$ when $x=0 ; C, C_{1}, C_{2}, \ldots$ will denote different positive constants whose exact values are not essential to the exposition of arguments.

The paper is organized as follows: in section 2 we present the framework's problem and some technical and essential results, mostly concerning the associated functional. Section 3 consists in the proof of a key proposition and our first main result. Finally, in section 4, we prove our second main result, in which we deal with the subcritical case and obtain multiplicity.

\section{Framework for Problem (1.1)}

In this section we establish the necessary framework for solving (1.1). For each $s \in(0,1)$ and $p>2$, we consider the Sobolev space $W^{s, p}\left(\mathbb{R}^{N}\right)=\left\{u \in L^{p}\left(\mathbb{R}^{N}\right) ;[u]_{s, p}<+\infty\right\}$, where

$$
[u]_{s, p}^{p}=\int_{\mathbb{R}^{N}} \int_{\mathbb{R}^{N}} \frac{|u(x)-u(y)|^{p}}{|x-y|^{N+p s}} d x d y
$$

is the Gagliardo seminorm. It is well-known that the space $\left(W^{s, p}\left(\mathbb{R}^{N}\right),\|\cdot\|\right)$, where $\|\cdot\|^{p}=$ $[\cdot]_{s, p}^{p}+\|\cdot\|_{p}^{p}$, is an uniformly convex Banach space, particularly reflexive, and separable (see [23, Theorem A.3]).

Next, we recall that, for a function $u \in C_{0}^{\infty}\left(\mathbb{R}^{N}\right)$, the fractional $p$-Laplacian operator $(-\Delta)_{p}^{s}$ is given by

$$
(-\Delta)_{p}^{s} u(x)=C(N, s) \lim _{\varepsilon \rightarrow 0} \int_{\mathbb{R}^{N} \backslash B_{\varepsilon}(x)} \frac{|u(x)-u(y)|^{p-2}(u(x)-u(y))}{|x-y|^{N+p s}} d y, \forall x \in \mathbb{R}^{N},
$$

where the normalizing constant $C(N, s)$ is defined in [13]. Throughout this paper we omit the normalizing constant to simplify the expressions. We also remember the reader that $C_{0}^{\infty}\left(\mathbb{R}^{N}\right)$ is dense in $W^{s, p}\left(\mathbb{R}^{N}\right)$ (see [1, Theorem 7.38]). For further considerations about $W^{s, p}\left(\mathbb{R}^{N}\right)$ and $(-\Delta)_{p}^{s}$ and some useful results, we refer to $[1,3,12,13,21,22]$.

We recall that $u \in W^{s, p}\left(\mathbb{R}^{N}\right)$ is a weak solution for (1.1) if

$$
\begin{aligned}
& \int_{\mathbb{R}^{N}} \int_{\mathbb{R}^{N}} \frac{|u(x)-u(y)|^{p-2}(u(x)-u(y))(v(x)-v(y))}{|x-y|^{N+s p}} d x d y+a \int_{\mathbb{R}^{N}}|u|^{p-2} u v d x \\
& +\int_{\mathbb{R}^{N}} \int_{\mathbb{R}^{N}} \ln (|x-y|)|u(x)|^{p}|u(y)|^{p-2} u(y) v(y) d x d y=\int_{\mathbb{R}^{N}} f(u) v d x, \forall v \in W^{s, p}\left(\mathbb{R}^{N}\right) .
\end{aligned}
$$

In this sense, we consider the associated functional $I: W^{s, p}\left(\mathbb{R}^{N}\right) \rightarrow \mathbb{R} \cup\{+\infty\}$ given by

$$
I(u)=\frac{1}{p}|| u \|^{p}+\frac{1}{2 p} \int_{\mathbb{R}^{N}} \int_{\mathbb{R}^{N}} \ln (|x-y|)|u(x)|^{p}|u(y)|^{p} d x d y-\int_{\mathbb{R}^{N}} F(u) d x .
$$

Then, as we will see, critical points of $I$ will be weak solutions for (1.1). However, one can see that $I$ is not well-defined over the whole space $W^{s, p}\left(\mathbb{R}^{N}\right)$, since we have the term involving the 
logarithmic function. Hence, following the ideas introduced by Stubbe [34], we consider the slightly smaller space

$$
X=\left\{u \in W^{s, p}\left(\mathbb{R}^{N}\right) ; \int_{\mathbb{R}^{N}} \ln (1+|x|)|u(x)|^{p} d x<+\infty\right\} .
$$

It is possible to verify that

$$
\|u\|_{*}=\int_{\mathbb{R}^{N}} \ln (1+|x|)|u(x)|^{p} d x
$$

defines a norm in $X$. The next natural step is to guarantee that $X$ enjoys of all good properties that we need. So, we start defining a measure $\eta: \mathcal{L} \rightarrow[-\infty,+\infty]$ given by

$$
\eta(E)=\int_{E} \ln (1+|x|)|u(x)|^{p} d x
$$

where $\mathcal{L}$ is the Lebesgue $\sigma$-algebra in $\mathbb{R}^{N}$. Then, we can consider the measure space $L^{p}\left(\mathbb{R}^{N}, \mathcal{L}, \eta\right)$ and, for each $u: \mathbb{R}^{N} \rightarrow \mathbb{R}$ Lebesgue measurable, we have $\|u\|_{L^{p\left(\mathbb{R}^{N}, \mathcal{L}, \eta\right)}}=\|u\|_{*}$.

Consequently, from Riesz-Fischer Theorem (see Folland [18]), the definition of $L^{p}$ spaces and the fact that $X=W^{s, p}\left(\mathbb{R}^{N}\right) \cap L^{p}\left(\mathbb{R}^{N}, \mathcal{L}, \eta\right)$, we conclude that $X$ is a Banach space endowed with the norm $\|\cdot\|_{X}^{p}=\|\cdot\|^{p}+\|\cdot\|_{*}^{p}$. Moreover, as [3, Proposition A.6], one can show that $X$ is uniformly convex. Hence, from [7, Theorem 3.31], $X$ is reflexive.

The following lemma plays a key role on the continuity of the functional $I$ and allow us to verify that $I$ is lower semicontinuous for $W^{s, p}\left(\mathbb{R}^{N}\right)$ and weakly lower semicontinuous for $X$. In order to do that, we need a result in order to control the exponential term.

Lemma 2.1. Let $\left(\varphi_{n}\right) \subset X$ and $\varphi \in X$. Then,

(a) if $\varphi_{n} \rightarrow \varphi$ in $W^{s, p}\left(\mathbb{R}^{N}\right)$ or $\varphi_{n} \rightarrow \varphi$ in $X$, then there exists a subsequence $\left(\varphi_{n_{k}}\right) \subset\left(\varphi_{n}\right)$ and a function $h \in W^{s, p}\left(\mathbb{R}^{N}\right)$ such that $\varphi_{n_{k}}(x) \rightarrow \varphi(x)$ a.e. in $\mathbb{R}^{N}$ and $\left|\varphi_{n_{k}}(x)\right| \leq h(x)$, for all $k \in \mathbb{N}$ and a.e. in $\mathbb{R}^{N}$.

(b) if $\varphi_{n} \rightarrow \varphi$ in $X$, then there exists a subsequence $\left(\varphi_{n_{k}}\right) \subset\left(\varphi_{n}\right)$ and a function $h \in X$ such that $\varphi_{n_{k}}(x) \rightarrow \varphi(x)$ a.e. in $\mathbb{R}^{N}$ and $\left|\varphi_{n_{k}}(x)\right| \leq h(x)$, for all $k \in \mathbb{N}$ and a.e. in $\mathbb{R}^{N}$.

Proof. We will use the construction done in [14, Proposition 2.7]. For items (a) and (b), similarly as [5, Lemma 2.4], one can see that the function $w$ constructed in [14] belongs to $W^{s, p}\left(\mathbb{R}^{N}\right)$. Here, we only highlight, why in item (b) we have $w \in X$.

By construction, one has $\left\|w_{n}\right\|_{*} \leq 1$, for all $n \in \mathbb{N}$. Since $w_{n+1}(x) \geq w_{n}(x)$ and $w_{n}(x) \rightarrow$ $w(x)$ a.e. in $\mathbb{R}^{N}$, follows $\ln (1+|x|)\left|g_{j}(x)\right|^{p} \rightarrow \ln (1+|x|)|g(x)|^{p}$ a.e. in $\mathbb{R}^{N}$ and, from the Monotone Convergence Theorem,

$$
\int_{\mathbb{R}^{N}} \ln (1+|x|)|w(x)|^{p} d x=\lim \int_{\mathbb{R}^{N}} \ln (1+|x|)\left|w_{n}(x)\right|^{p} d x \leq 1<+\infty .
$$

Therefore, $w \in X$. 
We would like to call attention that, essentially, the above lemma tell us that the obtained function $h$ satisfies $\|h\|_{*}<+\infty$. It will be very important in order to verify that $I$ is $C^{1}$.

To finish this part, we consider the operators $A: W^{s, p}\left(\mathbb{R}^{N}\right) \rightarrow\left(W^{s, p}\left(\mathbb{R}^{N}\right)\right)^{*}$ given by

$$
A(u)(v)=\int_{\mathbb{R}^{N}} \int_{\mathbb{R}^{N}} \frac{|u(x)-u(y)|^{p-2}(u(x)-u(y))(v(x)-v(y))}{|x-y|^{N+s p}} d x d y, \forall u, v \in X
$$

and $\tilde{A}: W^{s, p}\left(\mathbb{R}^{N}\right) \rightarrow\left(W^{s, p}\left(\mathbb{R}^{N}\right)\right)^{*}$ defined as

$$
\tilde{A}(u)(v)=A(u)(v)+\int_{\mathbb{R}^{N}} a|u|^{p-2} u v d x, \forall u, v \in X
$$

One can easily verify that $\tilde{A}(u)(v) \leq\|u\|^{p-1}\|v\|$ and $\tilde{A}(u)(u)=\|u\|^{p}$, for all $u, v \in W^{s, p}\left(\mathbb{R}^{N}\right)$. Therefore, as $W^{s, p}\left(\mathbb{R}^{N}\right)$ is uniformly convex, from [32, Proposition 1.3], $\tilde{A}$ verifies the $(S)$ property, that is, for any sequence $\left(u_{n}\right) \subset W^{s, p}\left(\mathbb{R}^{N}\right)$ satisfying $u_{n} \rightarrow u$ in $W^{s, p}\left(\mathbb{R}^{N}\right)$ and $\tilde{A}\left(u_{n}\right)\left(u_{n}-u\right) \rightarrow 0$, there exists a subsequence, still denoted by $\left(u_{n}\right)$, such that $u_{n} \rightarrow u$ in $W^{s, p}\left(\mathbb{R}^{N}\right)$.

For the second half of this section, we present some embedding results for $X$ and verify that $I$ is well-defined over $X$ and of class $C^{1}$. Clearly, $X \hookrightarrow W^{s, p}\left(\mathbb{R}^{N}\right)$, once $\|\cdot\| \leq\|\cdot\|_{X}$. Moreover, as the proof of the compactness embedding can be done similarly as [5, Lemma 2.1], we omit it here.

Proposition 2.1. The space $X$ is compactly embedded in $L^{\omega}\left(\mathbb{R}^{N}\right)$, for all $\omega \geq p$.

Before our next result, we recall the celebrated Moser-Trudinger Lemma for unbounded domains and a very useful lemma concerning $R(\alpha, t)$.

Lemma 2.2. (Moser-Trudinger Lemma [37, Theorem 1.1]) Let $s \in(0,1)$ and $s p=N$. Then, there exists $\alpha_{s, N}^{*}>0$ such that for every $0 \leq \alpha<\alpha_{s, N}^{*}$, the following inequality holds

$$
\sup _{u \in W^{s, p}\left(\mathbb{R}^{N}\right),\|u\| \leq 1} \int_{\mathbb{R}^{N}} R(\alpha, u) d x<+\infty .
$$

Lemma 2.3. ( [27, Lemma 2.3]) Let $\alpha>0$ and $r>1$. Then, for every $\beta>r$, there exists $a$ constant $C_{\beta}=C(\beta)>0$ such that

$$
\left(\exp \left(\alpha|t|^{p^{\prime}}\right)-S_{k_{p}-2}(\alpha, t)\right)^{r} \leq C_{\beta}\left(\exp \left(\beta \alpha|t|^{p^{\prime}}-S_{k_{p}-2}(\beta \alpha, t)\right)\right.
$$

with $\frac{1}{p}+\frac{1}{p^{\prime}}=1$.

Lemma 2.4. Let $\alpha>0$. Then, $R(\alpha, u) \in L^{1}\left(\mathbb{R}^{N}\right)$, for all $u \in W^{s, p}\left(\mathbb{R}^{N}\right)$.

Proof. Let $u \in W^{s, p}\left(\mathbb{R}^{N}\right) \backslash\{0\}$ and $\varepsilon>0$. Since $C_{0}^{\infty}\left(\mathbb{R}^{N}\right)$ is dense in $W^{s, p}\left(\mathbb{R}^{N}\right)$, there exists $\phi \in C_{0}^{\infty}\left(\mathbb{R}^{N}\right)$ such that $\|u-\phi\|<\varepsilon$. Observe that, for each $k \geq k_{p}-1$,

$$
|u|^{\frac{N}{N-s} k} \leq 2^{\frac{N}{N-s} k} \varepsilon^{\frac{N}{N-s} k}\left|\frac{u-\phi}{\|u-\phi\|}\right|^{\frac{N}{N-s} k}+2^{\frac{N}{N-s} k}|\phi|^{\frac{N}{N-s} k} .
$$


Consequently,

$$
R(\alpha, u) \leq R\left(\alpha 2^{\frac{N}{N-s}} \varepsilon^{\frac{N}{N-s}},\left|\frac{u-\phi}{\|u-\phi\|}\right|^{\frac{N}{N-s}}\right)+R\left(\alpha 2^{\frac{N}{N-s}},|\phi|^{\frac{N}{N-s}}\right) .
$$

From Lemma 2.2, choosing $\varepsilon>0$ sufficiently small such that $\alpha 2^{\frac{N}{N-s}} \varepsilon^{\frac{N}{N-s}}<\alpha_{s, N}^{*}$, we have

$$
\int_{\mathbb{R}^{N}} R\left(\alpha 2^{\frac{N}{N-s}} \varepsilon^{\frac{N}{N-s}},\left|\frac{u-\phi}{\|u-\phi\|}\right|^{\frac{N}{N-s}}\right) d x<+\infty .
$$

On the other side, since $\exp \left(\alpha 2^{\frac{N}{N-s}}|\phi|^{\frac{N}{N-s}}\right)=\sum_{k=0}^{+\infty} \frac{\alpha^{k}}{k !} 2^{\frac{N}{N-s} k}|\phi|^{\frac{N}{N-s} k}$, there exists $k_{0} \in \mathbb{N}$ such that $\sum_{k=k_{0}}^{+\infty} \frac{\alpha^{k}}{k !} 2^{\frac{N}{N-s} k}|\phi|^{\frac{N}{N-s} k}<\varepsilon$. This fact, combined with the fact that $\frac{N}{N-s} k>0$ for all $k_{p}-1 \leq$ $k \leq k_{0}$, give us

$$
\int_{\mathbb{R}^{N}} R\left(\alpha 2^{\frac{N}{N-s}},|\phi|^{\frac{N}{N-s}}\right) d x=\int_{\text {supp } \phi} R\left(\alpha 2^{\frac{N}{N-s}},|\phi|^{\frac{N}{N-s}}\right) d x<+\infty .
$$

Therefore, $R(\alpha, u) \in L^{1}\left(\mathbb{R}^{N}\right)$, for all $u \in W^{s, p}\left(\mathbb{R}^{N}\right)$.

Remark 2.1. From Lemmas 2.3 and 2.4, we conclude that $R(\alpha, u)^{l} \in L^{1}\left(\mathbb{R}^{N}\right)$, for all $u \in$ $W^{s, p}\left(\mathbb{R}^{N}\right), \alpha>0$ and $l \geq 1$.

Remark 2.2. Given $\varepsilon>0$, from $\left(f_{3}\right)$, there exists $\delta>0$ such that $|f(u)| \leq \varepsilon|u|^{p-1}$, for all $|u| \leq \delta$. Now, as $q>p$, there exists $r>0$ such that $q=p+r$. So, once $z^{q-1}, z^{\frac{N}{N-s} k}$, for all $k \geq k_{p}-1$, and $z^{r}$ are increasing functions, for $|u| \geq \delta$,

$$
|F(u)| \leq \frac{\varepsilon}{p}|u|^{p}+b_{1}|u| R(\alpha, u) \leq \frac{\varepsilon}{p}|u|^{p} \frac{|u|^{r} R(\alpha, u)}{\delta^{r} R(\alpha, \delta)}+b_{1}|u| \frac{|u|^{q-1}}{\delta^{q-1}} R(\alpha, u)=b_{2}|u|^{q} R(\alpha, u),
$$

where $b_{2}=\frac{\varepsilon}{p \delta^{r} R(\alpha, \delta)}+\frac{b_{1}}{\delta^{q-1}}>0$. Therefore, for $\alpha>\alpha_{0}$,

$$
|F(u)| \leq \frac{\varepsilon}{p}|u|^{p}+b_{2}|u|^{q} R(\alpha, u), \forall u \in X .
$$

Let $t, t^{\prime}>1$, with $\frac{1}{t}+\frac{1}{t^{\prime}}=1$. From (2.3), Remark 2.1, $X \hookrightarrow W^{s, p}\left(\mathbb{R}^{N}\right) \hookrightarrow L^{\omega}\left(\mathbb{R}^{N}\right)$, for all $\omega \geq p$, and Hölder inequality, we conclude that

$$
\int_{\mathbb{R}^{N}}|F(u)| d x \leq \frac{\varepsilon}{p}\|u\|_{p}^{p}+b_{2}\|u\|_{q t}^{q}\left(\int_{\mathbb{R}^{N}} R(\alpha, u)^{t^{\prime}} d x\right)^{\frac{1}{t^{\prime}}}<+\infty, \forall u \in X .
$$

Remark 2.3. Analogously as in Remark 2.2, for every $\varepsilon>0, \alpha>\alpha_{0}, q>p$ and $u \in X$, one can obtain

$$
|f(u)| \leq \varepsilon|u|^{p-1}+b_{1}|u|^{q-1} R(\alpha, u)
$$


Next, inspired by [10], we define three auxiliar functionals $V_{1}: W^{s, p}\left(\mathbb{R}^{N}\right) \rightarrow[0, \infty], V_{2}$ : $L^{\frac{2 N}{2 N-1} p}\left(\mathbb{R}^{N}\right) \rightarrow[0, \infty)$ and $V_{0}: W^{s, p}\left(\mathbb{R}^{N}\right) \rightarrow \mathbb{R} \cup\{\infty\}$, given by

$$
\begin{gathered}
u \mapsto V_{1}(u)=\int_{\mathbb{R}^{N}} \int_{\mathbb{R}^{N}} \ln (1+|x-y|)|u(x)|^{p}|u(y)|^{p} d x d y, \\
u \mapsto V_{2}(u, v)=\int_{\mathbb{R}^{N}} \int_{\mathbb{R}^{N}} \ln \left(1+\frac{1}{|x-y|}\right)|u(x)|^{p}|u(y)|^{p} d x d y, \\
u \mapsto V_{0}(u, v)=V_{1}(u, v)-V_{2}(u, v)=\iint_{\mathbb{R}^{N}} \ln (|x-y|)|u(x)|^{p}|u(y)|^{p} d x d y .
\end{gathered}
$$

These definitions are understood to being over measurable function $u, v: \mathbb{R}^{N} \rightarrow \mathbb{R}$, such that the integrals are defined in the Lebesgue sense.

Remark 2.4. (i) As a consequence of Hardy-Littlewood-Sobolev Inequality (HLS) [29], with $\alpha=\beta=0$ and $\lambda=1$, we have $\frac{1}{q}+\frac{1}{t}+\frac{1}{N}=2$. So, making a natural choice for $q$ and $t$, that is $q=t=\frac{2 N}{2 N-1}$, we obtain

$$
\left|V_{2}(u)\right| \leq K_{0}\|u\|_{\frac{2 N}{2 N-1} p}^{2 p}, \quad \forall u \in L^{\frac{2 N}{2 N-1} p}\left(\mathbb{R}^{N}\right)
$$

so $V_{2}$ takes finite values over $L^{\frac{2 N}{2 N-1} p}\left(\mathbb{R}^{N}\right)$.

(ii) $V_{1}(u) \leq 2|| u||_{*}^{p}|| u \|_{p}^{p}$, since $\ln (1+|x-y|) \leq \ln (1+|x|)+\ln (1+|y|)$.

(iii) $\int_{\mathbb{R}^{N}} \int_{\mathbb{R}^{N}} \ln (1+|x-y|)|u(x)|^{p}|v(y)|^{p} d x d y \leq\left.\|u\|_{*}^{p}\|v\|\right|_{p} ^{p}+\|v\|_{*}^{p}\|u\|_{p}^{p}$.

Lemma 2.5. Let $\left(u_{n}\right) \subset X$ and $u \in X$ such that $u_{n} \rightarrow u$ on $W^{s, p}\left(\mathbb{R}^{N}\right)$. Then, we have

$$
\int_{\mathbb{R}^{N}} F\left(u_{n}\right) \rightarrow \int_{\mathbb{R}^{N}} F(u), \int_{\mathbb{R}^{N}} f\left(u_{n}\right) u_{n} \rightarrow \int_{\mathbb{R}^{N}} f(u) u \text { and } \int_{\mathbb{R}^{N}} f\left(u_{n}\right) v \rightarrow \int_{\mathbb{R}^{N}} f(u) v, \forall v \in X
$$

Proof. Since $u_{n} \rightarrow u$ in $W^{s, p}\left(\mathbb{R}^{N}\right), u_{n}(x) \rightarrow u(x)$ a.e. in $\mathbb{R}$ and, from [13, Theorem 6.9], $u_{n} \rightarrow u$ in $L^{\omega}\left(\mathbb{R}^{N}\right)$ for all $\omega \geq p$. By Lemma 2.1 and using the Dominated Convergence Theorem, the result follows.

One can see, from (2.4) and Remark 2.4, that $I(u)<+\infty$ for all $u \in X$.

Lemma 2.6. The functionals $V_{1}, V_{2}, V_{0}, I$ are of class $C^{1}(X, \mathbb{R})$, with

$$
V_{1}^{\prime}(u)(v)=2 p \int_{\mathbb{R}^{N}} \int_{\mathbb{R}^{N}} \ln (1+|x-y|)|u(x)|^{p}|u(y)|^{p-2} u(y) v(y) d x d y .
$$

and

$$
V_{2}^{\prime}(u)(v)=2 p \int_{\mathbb{R}^{N}} \int_{\mathbb{R}^{N}} \ln \left(1+\frac{1}{|x-y|}\right)|u(x)|^{p}|u(y)|^{p-2} u(y) v(y) d x d y .
$$


Proof. The proof follows by a standard way, combining the Mean Value Theorem with Lemma 2.1, for $V_{1}$ and $V_{2}$, and by Lemma 2.5 and [22, Lemma 2].

Remark 2.5. Note that, if $u \in X$, then $u \in L^{\frac{2 N}{2 N-1} p}\left(\mathbb{R}^{N}\right)$. So, one can see that $V_{2}$ is of class $C^{1}$ in $L^{\frac{2 N}{2 N-1} p}\left(\mathbb{R}^{N}\right)$.

Lemma 2.7. (i) The functional $V_{1}$ is weakly lower semicontinuous in $W^{s, p}\left(\mathbb{R}^{N}\right)$.

(ii) The functional I is weakly lower semicontinuous in X.

(iii) The functional I is lower semicontinuous in $W^{s, p}\left(\mathbb{R}^{N}\right)$.

Proof. (i) Let $\left(u_{n}\right) \subset W^{s, p}\left(\mathbb{R}^{N}\right)$ such that $u_{n} \rightarrow u$. Then, $u_{n} \rightarrow u$ in $W^{s, p}\left(B_{R}\right)$ and, from [13, Theorem 7.1], $u_{n} \rightarrow u$ in $L^{p}\left(B_{R}\right)$, for all $R>0$. We claim that

$$
\lim _{n \rightarrow+\infty} \int_{B_{R}} \int_{B_{R}} \ln (1+|x-y|)\left|u_{n}(x)\right|^{p}\left|u_{n}(y)\right|^{p} d x d y=\iint_{B_{R}} \int_{B_{R}} \ln (1+|x-y|)|u(x)|^{p}|u(y)|^{p} d x d y .
$$

Indeed, up to subsequence, one can see that $\left.\int_{B_{R}}|| u_{n}(y)\right|^{p}-|u(y)|^{p} \mid d y \rightarrow 0$. Then,

$$
\begin{aligned}
& \left.\left|\int_{B_{R}} \int_{B_{R}} \ln (1+|x-y|)\right| u_{n}(x)\right|^{p}\left|u_{n}(y)\right|^{p} d x d y-\int_{B_{R}} \int_{B_{R}} \ln (1+|x-y|)|u(x)|^{p}|u(y)|^{p} d x d y \mid \\
& \leq\left[\ln (1+2 R)|| u_{n}\left\|_{p}^{p}+\ln (1+2 R)|| u\right\|_{p}^{p}\right] \int_{B_{R}} \|\left. u_{n}(x)\right|^{p}-|u(x)|^{p} \mid d x \rightarrow 0 .
\end{aligned}
$$

As a consequence, for each $R>0$,

$$
\liminf V_{1}\left(u_{n}\right) \geq \int_{B_{R}} \int_{B_{R}} \ln (1+|x-y|)|u(x)|^{p}|u(y)|^{p} d x d y .
$$

Hence, from the Monotone Convergence Theorem,

$$
\liminf V_{1}\left(u_{n}\right) \geq \lim _{R \rightarrow+\infty} \int_{B_{R}} \int_{B_{R}} \ln (1+|x-y|)|u(x)|^{p}|u(y)|^{p} d x d y=V_{1}(u) .
$$

(ii) Follows from the fact that $V_{2} \in C^{1}\left(L^{\frac{2 N}{2 N-1} p}, \mathbb{R}\right),(2.6), X \hookrightarrow W^{s, p}\left(\mathbb{R}^{N}\right)$, Proposition 2.1 and item (i).

(iii) Follows from (2.6), [13, Theorem 6.9], item (i) and the fact that $I(u)-\frac{1}{2 p} V_{1}(u)$ is continuous with respect to $\|\cdot\|$.

\section{Geometry of $I$ and Technical Results}

In this section we will provide some technical results and investigate the geometry of $I$. First of all, we will verify some conditions that can allow us to get convergence in $X$. Then, we see how 
one can get boundedness for the exponential term. Finally, we verify that $I$ has the mountain pass geometry.

Lemma 3.1. Let $\left(u_{n}\right) \subset X$ such that $u_{n} \rightarrow u$ in $X$. Then,

$$
\lim _{n \rightarrow+\infty} \int_{\mathbb{R}^{N}} \int_{\mathbb{R}^{N}} \ln (1+|x-y|)\left|u_{n}(x)\right|^{p}|u(y)|^{p-2} u(y)\left(u_{n}(y)-u(y)\right) d x d y=0 .
$$

Proof. First of all, we have

$$
\begin{aligned}
& \left.\left|\int_{\mathbb{R}^{N}} \int_{\mathbb{R}^{N}} \ln (1+|x-y|)\right| u_{n}(x)\right|^{p}|u(y)|^{p-2} u(y)\left(u_{n}(y)-u(y)\right) d x d y \mid \\
& \leq\left\|u_{n}\right\|_{*}^{p}|| u\left\|_{p}^{p-1}|| u_{n}-u\right\|_{p}+\int_{\mathbb{R}^{N}} \int_{\mathbb{R}^{N}} \ln (1+|y|)\left|u_{n}(x)\right|^{p}|u(y)|^{p-1}\left|u_{n}(y)-u(y)\right| d x d y .
\end{aligned}
$$

Now, for any $R>0$, fixed, define

$$
\int_{\mathbb{R}^{N}} \ln (1+|y|)|u(y)|^{p-1}\left|u_{n}(y)-u(y)\right| d y=h_{n}(R)+g_{n}(R),
$$

where

$$
h_{n}(R)=\int_{B_{R}} \ln (1+|y|)|u(y)|^{p-1}\left|u_{n}(y)-u(y)\right| d y
$$

and

$$
g_{n}(R)=\int_{B_{R}^{c}} \ln (1+|y|)|u(y)|^{p-1}\left|u_{n}(y)-u(y)\right| d y .
$$

For $y \in B_{R}, \ln (1+|y|) \leq \ln (1+R)$. Since $u_{n} \rightarrow u$ in $X$, by Proposition $2.1, u_{n} \rightarrow u$ in $L^{\omega}\left(\mathbb{R}^{N}\right)$, for all $\omega \geq p$. Thus,

$$
\left|h_{n}(R)\right| \leq \ln (1+R)\|u\|_{p}^{p-1}|| u_{n}-u \|_{p} \rightarrow 0,
$$

as $n \rightarrow+\infty$. On the other hand,

$$
g_{n}(R) \leq\left(\int_{B_{R}^{c}} \ln (1+|y|)|u(y)|^{p} d y\right)^{\frac{1}{p^{\prime}}}\left(\int_{B_{R}^{c}} \ln (1+|y|)\left|u_{n}(y)-u(y)\right|^{p} d y\right)^{\frac{1}{p}} .
$$

Note that

$$
\left(\int_{B_{R}^{c}} \ln (1+|y|)\left|u_{n}(y)-u(y)\right|^{p} d y\right)^{\frac{1}{p}} \leq C_{1}\left(\left\|u_{n}\right\|_{*}+\|u\|_{*}\right) \leq C_{2},
$$


since $\left(u_{n}\right) \subset X$ is bounded. Then, from (3.1),

$$
g_{n}(R) \leq C_{1}\left(\int_{B_{R}^{c}} \ln (1+|y|)|u(y)|^{p} d y\right)^{\frac{1}{p^{\prime}}}=C_{1} \varphi(R) \rightarrow 0, \text { as } R \rightarrow+\infty
$$

Consequently, for all $R>0$,

$$
\left.\limsup _{n \rightarrow+\infty}\left|\int_{\mathbb{R}^{N}} \int_{\mathbb{R}^{N}} \ln (1+|x-y|)\right| u_{n}(x)\right|^{p}|u(y)|^{p-2} u(y)\left(u_{n}(y)-u(y)\right) d x d y \mid \leq C_{1} \varphi(R) .
$$

Thus, taking $R \rightarrow+\infty, \varphi(R) \rightarrow 0$ and we conclude the proof.

In order to prove the next proposition, we will need the following technical lemma, which is, essentially, a corollary of Ergorov's Theorem.

Lemma 3.2. Let $u \in L^{p}\left(\mathbb{R}^{N}\right) \backslash\{0\}$ and $\left(u_{n}\right) \subset L^{p}\left(\mathbb{R}^{N}\right)$ such that $u_{n}(x) \rightarrow u(x)$ a.e in $\mathbb{R}^{N}$. Then, there exists $R \in \mathbb{N}, \delta>0, n_{0} \in \mathbb{N}$ and $A \subset B_{R}$, such that $A$ is measurable, $\mu(A)>0$ and $u_{n}(x)>\delta$, for all $x \in A$ and for all $n \geq n_{0}$.

Proposition 3.1. Let $u \in L^{p}\left(\mathbb{R}^{N}\right) \backslash\{0\},\left(u_{n}\right) \subset L^{p}\left(\mathbb{R}^{N}\right)$ such that $u_{n}(x) \rightarrow u(x)$ a.e in $\mathbb{R}^{N}$ and $\left(v_{n}\right) \subset L^{p}\left(\mathbb{R}^{N}\right)$ bounded. If

$$
\alpha=\sup _{n} \int_{\mathbb{R}^{N}} \int_{\mathbb{R}^{N}} \ln (1+|x-y|)\left|u_{n}(x)\right|^{p}\left|v_{n}(y)\right|^{p} d x d y<+\infty
$$

then $\left\|v_{n}\right\|_{*}$ is bounded. Moreover, setting

$$
\alpha_{n}=\int_{\mathbb{R}^{N}} \int_{\mathbb{R}^{N}} \ln (1+|x-y|)\left|u_{n}(x)\right|^{p}\left|v_{n}(y)\right|^{p} d x d y
$$

for each $n \in \mathbb{N}$, if $\alpha_{n} \rightarrow 0$ and $\left\|v_{n}\right\|_{p} \rightarrow 0$, then $\left\|v_{n}\right\|_{*} \rightarrow 0$.

Proof. Let $n_{0}, R, \delta$ and $A$ is in Lemma 3.2. Then, $u_{n}(x)>\delta$, for all $n \geq n_{0}$. From $\ln$ properties and the fact that $\alpha_{n} \geq 0$, for all $n \in \mathbb{N}$, we have

$$
\begin{aligned}
\alpha_{n} & \geq \int_{B_{2 R}^{c}} \int_{A} \ln (1+|x-y|)\left|u_{n}(x)\right|^{p}|v(y)|^{p} d x d y \\
& >\delta^{p} \int_{B_{2 R}^{c}} \int_{A} \ln (\sqrt{1+|y|})\left|v_{n}(y)\right|^{p} d x d y \\
& =\frac{\delta^{p} \mu(A)}{2}\left(\int_{\mathbb{R}^{N}} \ln (1+|y|)\left|v_{n}(y)\right|^{p} d y-\int_{B_{2 R}} \ln (1+|y|)\left|v_{n}(y)\right|^{p} d y\right) \\
& \geq \frac{\delta^{p} \mu(A)}{2}\left(\|v\|_{*}^{p}-\ln (1+2 R)|| v_{n} \|_{p}^{p}\right) .
\end{aligned}
$$


Consequently,

$$
0 \leq\left\|v_{n}\right\|_{*} \leq\left(\frac{2}{\mu(A) \delta^{p}} \alpha_{n}+\ln (1+2 R)\left\|v_{n}\right\|_{p}^{p}\right)^{\frac{1}{p}} .
$$

Therefore, from hypothesis, $\left(\left\|v_{n}\right\|_{*}\right) \subset \mathbb{R}$ is bounded. Moreover, if $\alpha_{n} \rightarrow 0$ and $\left\|v_{n}\right\|_{p} \rightarrow 0$, from equation (3.3), $\left\|v_{n}\right\|_{*} \rightarrow 0$.

Lemma 3.3. Let $u \in W^{s, p}\left(\mathbb{R}^{N}\right), r>p, l \geq 1, \beta>0$ and $\|u\| \leq M$, for $M>0$ sufficiently small. Then, there exists a constant $K_{1}=K_{1}(\beta, N, M, l, s)>0$ such that

$$
\int_{\mathbb{R}^{N}}|u|^{r} R(\beta, u)^{l} d x \leq K_{1}\|u\|_{t_{0}}^{r}
$$

for some $t_{0}>p$.

Proof. From Lemma 2.3, for $\beta_{1}=\beta_{1}(l)>l$ with $\beta_{1} \sim l$, there exists a constant $C_{1}=C_{1}\left(\beta_{1}\right)>$ 0 such that $R(\beta, u)^{l} \leq C_{1} R\left(\beta_{1} \beta, u\right)$.

Let $t, t^{\prime}>1$ with $\frac{1}{t}+\frac{1}{t^{\prime}}=1$. From Hölder inequality,

$$
\int_{\mathbb{R}^{N}}|u|^{r} R(\beta, u)^{l} d x \leq C_{1} \int_{\mathbb{R}^{N}}|u|^{r} R\left(\beta_{1} \beta, u\right) d x \leq C_{1}\left(\int_{\mathbb{R}^{N}} R\left(\beta_{1} \beta, u\right)^{t} d x\right)^{\frac{1}{t}}\|u\|_{r t^{\prime}}^{r} .
$$

Once again, from Lemma 2.3, for $\beta_{2}=\beta_{2}(t)>t$ with $\beta_{2} \sim t$, there exists a constant $C_{2}=$ $C_{2}\left(\beta_{2}\right)>0$ satisfying $R\left(\beta_{1} \beta, u\right)^{t} \leq C_{2} R\left(\beta_{2} \beta_{2} \beta, u\right)$. Thus,

$$
\int_{\mathbb{R}^{N}}|u|^{r} R(\beta, u)^{l} d x \leq C_{1} C_{2}^{\frac{1}{t}}\left(\int_{\mathbb{R}^{N}} R\left(\beta_{2} \beta_{1} \beta, u\right) d x\right)^{\frac{1}{t}}\|u\|_{r t^{\prime}}^{r} .
$$

Write $R\left(\beta_{2} \beta_{1} \beta, u\right)=R\left(\beta_{2} \beta_{1} \beta\|u\|^{\frac{N}{N-s}}, \frac{u}{\|u\|}\right)$. Then, choosing $M>0$ sufficiently small such that $\beta_{2} \beta_{1} \beta\|u\|^{\frac{N}{N-s}}<\alpha_{s, N}^{*}$, from Lemma 2.2, we can find a constant $K_{1}=K_{1}(\beta, N, M, l, s)>$ 0 satisfying

$$
\int_{\mathbb{R}^{N}}|u|^{r} R(\beta, u)^{l} d x \leq K_{1}\|u\|_{r t^{\prime}}^{r}
$$

Setting $t_{0}=r t^{\prime}>p$, we have the desired result.

Remark 3.1. Under the hypothesis of Lemma 3.3, from the embeddings [13, Theorem 6.9] $t_{0}>p$, there exists a constant $K_{2}=K_{2}(\beta, N, M, l, s)>0$ such that

$$
\int_{\mathbb{R}^{N}}|u|^{r} R(\beta, u)^{l} d x \leq K_{2}\|u\|^{r}
$$

Moreover, since $\|\cdot\| \leq\|\cdot\|_{X}$, we have

$$
\int_{\mathbb{R}^{N}}|u|^{r} R(\beta, u)^{l} d x \leq K_{2}\|u\|_{X}^{r}
$$


Remark 3.2. Observe that, the estimates obtained in Lemma 3.3 and Remark 3.1 can be applied for an arbitrary, but fixed, $u \in X \backslash\{0\}$ making $\beta>0$ sufficiently small in order to apply MoserTrudinger Lemma 2.2.

Lemma 3.4. There exists $\rho>0$ such that

$$
m_{\beta}=\inf \{I(u) ; u \in X,\|u\|=\beta\}>0, \forall \beta \in(0, \rho]
$$

and

$$
n_{\beta}=\inf \left\{I^{\prime}(u)(u) ; u \in X,\|u\|=\beta\right\}>0, \forall \beta \in(0, \rho] .
$$

Proof. Let $u \in X \backslash\{0\}$, with $\|u\|$ sufficiently small in order to apply Lemma 3.3, and $q>p$. Then, from (2.6), Remark 3.1, (2.3) and Sobolev embeddings [13, Theorem 6.9], we have

$$
I(u) \geq \frac{1}{p}\|u\|^{p}-\frac{K_{0}}{2 p}\|u\|_{\frac{2 N}{2 N-1} p}^{2 p}-\frac{\varepsilon}{p}\|u\|_{p}^{p}-K_{2}\|u\|^{q} \geq \frac{\|u\|^{p}}{p}\left[1-\varepsilon-C_{1}\|u\|^{p}-C_{2}\|u\|^{q-p}\right] .
$$

Hence, for $\varepsilon>0$ and $\rho>0$ sufficiently small, we obtain (3.4). Similarly, from (2.5), (2.6), Remark 3.1 and Sobolev embeddings [13, Theorem 6.9], follows

$$
I^{\prime}(u)(u)=\|u\|^{p}+V_{1}(u)-V_{2}(u)-\int_{\mathbb{R}^{N}} f(u) u d x \geq\|u\| \|^{p}\left[1-\varepsilon-C_{3}\|u\|^{p}-C_{4}\|u\|^{q-p}\right] .
$$

Therefore, taking $\varepsilon, \rho>0$ sufficiently small, we get (3.5).

Lemma 3.5. Let $u \in X \backslash\{0\}, t>0$ and $q>2 p$. Then,

$$
\lim _{t \rightarrow 0} I(t u)=0, \sup _{t>0} I(t u)<+\infty \text { and } I(t u) \rightarrow-\infty \text { as } t \rightarrow+\infty .
$$

Proof. Let $u \in X \backslash\{0\}$. First of all, from $\left(f_{4}\right)$,

$$
I(t u)=\frac{t^{p}}{p}\|u\|^{p}+\frac{t^{2 p}}{2 p} V_{0}(u)-\int_{\mathbb{R}^{N}} F(t u) d x \leq \frac{t^{p}}{p}\|u\|^{p}+\frac{t^{2 p}}{2 p} V_{0}(u)-C_{q} t^{q}\|u\|_{q}^{q} \rightarrow-\infty
$$

as $t \rightarrow+\infty$. Now, from (2.3) and Lemma 3.3, for $t>0$ sufficiently small such that $\|t u\|$ is under Lemma 3.3 conditions, we have

$$
\left|\int_{\mathbb{R}^{N}} F(t u) d x\right| \leq \frac{t^{p}}{p}\|u\|^{p}+K_{1} t^{q}\|u\|_{t_{0}}^{q} \rightarrow 0
$$

as $t \rightarrow 0$. Hence, $I(t u) \rightarrow 0$ as $t \rightarrow 0$. Finally, once $I \in C^{1}(X, \mathbb{R})$, from the above two facts we conclude that $\sup _{t>0} I(t u)<+\infty$.

Consider a sequence $\left(u_{n}\right) \subset X$ satisfying

$\exists d>0$ s.t. $I\left(u_{n}\right)<d, \forall n \in \mathbb{N}$ and $\left\|I^{\prime}\left(u_{n}\right)\right\|_{X^{\prime}}\left(1+\left\|u_{n}\right\|_{X}\right) \rightarrow 0$, as $n \rightarrow+\infty$. 
Lemma 3.6. Let $\left(u_{n}\right) \subset X$ satisfying (3.6). Then, $\left(u_{n}\right)$ is bounded in $W^{s, p}\left(\mathbb{R}^{N}\right)$.

Proof. From (3.6) and $\left(f_{3}\right)$, we have

$$
d+o(1) \geq I\left(u_{n}\right)-\frac{1}{2 p} I^{\prime}\left(u_{n}\right)\left(u_{n}\right) \geq \frac{1}{2 p}\left\|u_{n}\right\|^{p}+\left(\frac{\theta}{2 p}-1\right) \int_{\mathbb{R}^{N}} F\left(u_{n}\right) d x \geq \frac{1}{2 p}\left\|u_{n}\right\|^{p},
$$

for all $n \in \mathbb{N}$. Therefore, $2 p d+o(1) \geq\left\|u_{n}\right\|^{p}$, for all $n \in \mathbb{N}$, and the lemma follows.

Remark 3.3. (1) Observe that exchanging the condition $I\left(u_{n}\right) \leq d$, for all $n \in \mathbb{N}$, for $I\left(u_{n}\right) \rightarrow$ $d>0$ the above result remains valid.

(2) One can easily verify that the value $c_{m p}$ satisfies $0<m_{\rho} \leq c_{m p}<+\infty$.

(3) Since I has the mountain pass geometry and $c_{m p}>0$, one can prove, as in [5], that there exists a sequence $\left(u_{n}\right) \subset X$ such that

$$
I\left(u_{n}\right) \rightarrow c_{m p} \quad \text { and } \quad\left\|I^{\prime}\left(u_{n}\right)\right\|_{X^{\prime}}\left(1+\left\|u_{n}\right\|_{X}\right) \rightarrow 0 .
$$

Moreover, such sequence satisfies (3.6).

Lemma 3.7. Let $\left(u_{n}\right) \subset X$ satisfying (3.7) and $q>2 p$. Then, for some $\rho_{0}>0$ sufficiently small,

$$
\limsup _{n}\left\|u_{n}\right\|^{p}<\rho_{0}^{p}
$$

Proof. From Lemma 3.6, $2 p c_{m p}+o(1) \geq\left\|u_{n}\right\|^{p}$, for all $n \in \mathbb{N}$. Then, $\lim \sup \left\|u_{n}\right\|^{p} \leq 2 p c_{m p}$. So, the natural step consists in finding a estimative for $c_{m p}$.

Consider the set $\mathcal{A}=\left\{u \in X \backslash\{0\} ; V_{0}(u) \leq 0\right\}$. For each $u \in X \backslash\{0\}, t>0$ and $x \in \mathbb{R}^{N}$, we define $u_{t}(x)=t^{2} u(t x)$. Then, a directly computation give us

$$
V_{0}\left(u_{t}\right)=t^{4 p-2 N} V_{0}(u)-t^{4 p-2 N} \ln t\|u\|_{p}^{2 p} \rightarrow-\infty,
$$

as $t \rightarrow+\infty$, since $4 p-2 N=2 p(2-s)>0$. Hence, $\mathcal{A} \neq \emptyset$.

Moreover, from the embeddings [13, Theorem 6.9], there exists $C>0$ such that $\|u\| \geq$ $C\|u\|_{q}$. So, it makes sense to define

$$
S_{q}(v)=\frac{\|v\|}{\|v\|_{q}} \quad \text { and } \quad S_{q}=\inf _{v \in \mathcal{A}} S_{q}(v) \geq \inf _{v \neq 0} S_{q}(v)>0 .
$$

Now, from Lemma 3.5, for $v \in \mathcal{A}$ and $T>0$ sufficiently large, $I(T v)<0$. Defining $\gamma:[0,1] \rightarrow X$ by $\gamma(t)=t T v$, we have that $\gamma \in \Gamma$ and

$$
c_{m p} \leq \max _{0 \leq t \leq 1} I(\gamma(t))=\max _{0 \leq t \leq 1} I(t T v) \leq \max _{t \geq 0} I(t v) .
$$

Consequently, for $\psi \in \mathcal{A}$,

$$
c_{m p} \leq \max _{t \geq 0} I(t \psi) \leq \max _{t \geq 0}\left\{\frac{t^{p}}{2 p}\|\psi\|^{p}-C_{q} t^{q}\|\psi\|_{q}^{q}\right\} \leq\left(\frac{1}{p}-\frac{1}{q}\right) \frac{S_{q}(\psi)^{\frac{p q}{q-p}}}{\left(q C_{q}\right)^{\frac{p}{q-p}}} .
$$


Taking the infimum over $\psi \in \mathcal{A}$, we obtain

$$
\limsup _{n}\left\|u_{n}\right\|^{p} \leq \frac{2(q-p)}{q} \frac{S_{q}^{\frac{p q}{q-p}}}{\left(q C_{q}\right)^{\frac{p}{q-p}}} \leq \rho_{0}^{p}
$$

for $C_{q}>0$ sufficiently large.

Remark 3.4. From Lemma 3.7, one can see that, taking $C_{q}>0$ sufficiently large, we can make $\rho_{0}$ sufficiently small such that every result concerning the exponential term is valid for sequences satisfying $I\left(u_{n}\right) \rightarrow c_{m p}$ or $I\left(u_{n}\right) \leq c_{m p}$.

\section{Proof of Theorem 1.1}

In the present section, we finish the proof of Theorem 1.1. We start proving a key proposition that provides us with nontrivial critical points for $I$ in $X$. Then, it remains just to gather all the results inside the theorem.

Proposition 4.1. Let $\left(f_{1}\right)-\left(f_{4}\right), q>2 p$ and $\left(u_{n}\right) \subset X$ satisfying (3.7). Then, up to a subsequence, only one between the two alternatives is valid

(a) $\left\|u_{n}\right\| \rightarrow 0$ and $I\left(u_{n}\right) \rightarrow 0$, as $n \rightarrow+\infty$.

(b) There exists points $y_{n} \in \mathbb{Z}^{N}$ such that $\tilde{u}_{n}=y_{n} * u_{n} \rightarrow u$ in $X$, for a nontrivial critical point $u \in X$ of $I$.

Proof. From Lemma 3.6, $\left(u_{n}\right) \subset W^{s, p}\left(\mathbb{R}^{N}\right)$ is bounded. Suppose that (a) does not happen.

Claim 1: $\liminf _{n \rightarrow+\infty} \sup _{y \in \mathbb{Z}^{N}} \int_{B_{2}(y)}\left|u_{n}(x)\right|^{p} d x>0$.

Lets suppose the contrary. Then, from and easy adaptation Lion's Lemma [36, Lemma 2.4], $u_{n} \rightarrow 0$ in $L^{\omega}\left(\mathbb{R}^{N}\right)$, for all $\omega>p$. Thus, since $\frac{2 N}{2 N-1} p>p$, from (2.6), $V_{2}\left(u_{n}\right) \rightarrow 0$. Moreover, from (2.5) and Remark 3.1, we have

$$
\left|\int_{\mathbb{R}^{N}} f\left(u_{n}\right) u_{n} d x\right| \leq \varepsilon\left\|u_{n}\right\|_{p}^{p}+C_{1}\left\|u_{n}\right\|_{q t_{0}}^{q} \leq \varepsilon C_{2}+C_{1}\left\|u_{n}\right\|_{q t_{0}}^{q} \rightarrow 0,
$$

as $\varepsilon \rightarrow 0$ and $n \rightarrow+\infty$. Consequently,

$$
\left\|u_{n}\right\|^{p}+V_{1}\left(u_{n}\right)=I^{\prime}\left(u_{n}\right)\left(u_{n}\right)+V_{2}\left(u_{n}\right)+\int_{\mathbb{R}^{N}} f\left(u_{n}\right) u_{n} d x \rightarrow 0
$$

as $n \rightarrow+\infty$. So, from the non-negativeness, we have $\left\|u_{n}\right\| \rightarrow 0$ and $V_{1}\left(u_{n}\right) \rightarrow 0$ and, from the embeddings [13, Theorem 6.9], $\left\|u_{n}\right\|_{p} \rightarrow 0$ and $\left\|u_{n}\right\|_{q t_{0}} \rightarrow 0$. Finally, from (2.3), Remark 3.1 and the embeddings [13, Theorem 6.9], we conclude that $\int_{\mathbb{R}^{N}} F\left(u_{n}\right) d x \rightarrow 0$. Hence, $I\left(u_{n}\right) \rightarrow 0$, which is a contradiction, proving the claim. 
As a consequence, one can easily obtain, up to subsequence, $\left(y_{n}\right) \subset \mathbb{Z}^{N}$ such that $\left(y_{n} * u_{n}\right) \subset$ $X$ and $\tilde{u}_{n}=y_{n} * u_{n} \rightarrow u$ in $W^{s, p}\left(\mathbb{R}^{N}\right) \backslash\{0\}$. So, without loss of generality, we can assume that $\tilde{u}_{n}(x) \rightarrow u(x)$ a.e. in $\mathbb{R}^{N}$ and, from [13, Theorem 6.9], $\left(\tilde{u}_{n}\right)$ is bounded in $L^{\omega}\left(\mathbb{R}^{N}\right)$ for all $\omega \geq p$.

Now, observe that, since $\frac{2 N}{2 N-1} p, q t_{0}>p$, we have

$$
\begin{aligned}
V_{1}\left(\tilde{u}_{n}\right)=V_{1}\left(u_{n}\right) & =I^{\prime}\left(u_{n}\right)\left(u_{n}\right)+V_{2}\left(u_{n}\right)+\int_{\mathbb{R}^{N}} f\left(u_{n}\right) u_{n} d x-\left\|u_{n}\right\|^{p} \\
& \leq K_{0}\left\|u_{n}\right\|_{\frac{2 N}{2 N-1} p}^{2 p}+\varepsilon C_{2} C_{1}\left\|u_{n}\right\|_{q t_{0}}^{q}+o(1) \leq C_{3}+o(1)
\end{aligned}
$$

That is, $\sup V_{1}\left(\tilde{u}_{n}\right)<+\infty$. So, from Proposition 3.1, $\left(\left\|\tilde{u}_{n}\right\|_{*}\right)$ is bounded. Since $\left(\tilde{u}_{n}\right)$ is already bounded in $W^{s, p}\left(\mathbb{R}^{N}\right),\left(\tilde{u}_{n}\right)$ is bounded in $X$. Hence, from the reflexiveness of $X$, passing to a subsequence if necessary, $u \in X$ and $\tilde{u}_{n} \rightarrow u$ in $X$. Moreover, from Proposition 2.1, $\tilde{u}_{n} \rightarrow u$ in $L^{\omega}\left(\mathbb{R}^{N}\right)$, for all $\omega \geq p$.

Claim 2: $I^{\prime}\left(\tilde{u}_{n}\right)\left(\tilde{u}_{n}-u\right) \rightarrow 0$, as $n \rightarrow+\infty$.

First of all, observe that, by a change of variables, $I^{\prime}\left(\tilde{u}_{n}\right)\left(\tilde{u}_{n}-u\right)=I^{\prime}\left(u_{n}\right)\left(u_{n}-\left(-y_{n}\right) * u\right)$. Thus,

$$
\left|I^{\prime}\left(\tilde{u}_{n}\right)\left(\tilde{u}_{n}-u\right)\right|=\left|I^{\prime}\left(u_{n}\right)\left(u_{n}-\left(-y_{n}\right) * u\right)\right| \leq\left\|I^{\prime}\left(u_{n}\right)\right\|_{X^{\prime}}\left(\left\|u_{n}\right\|_{X}+\left\|\left(-y_{n}\right) * u\right\|_{X}\right) .
$$

Then, similarly as in [5], we first seek for an useful inequality for $\left\|\left(-y_{n}\right) * u\right\|_{X}$. If $\left|y_{n}\right| \rightarrow+\infty$, then, for $x \in \mathbb{R}^{N}$,

$$
\ln \left(1+\left|x-y_{n}\right|\right)-\ln \left(1+\left|y_{n}\right|\right)=\ln \left(\frac{1+\left|x-y_{n}\right|}{1+\left|y_{n}\right|}\right) \rightarrow 0, n \rightarrow+\infty .
$$

Therefore, there exists $C_{4}>0$ such that $\ln \left(1+\left|x-y_{n}\right|\right) \geq C_{4} \ln \left(1+\left|y_{n}\right|\right)$.

Now, suppose that $\left(y_{n}\right) \subset \mathbb{Z}^{N}$ converges to $y_{0} \in \mathbb{Z}^{N}$. Then, up to a subsequence, $y_{n} \equiv y_{0}$. Let $y_{0} \neq 0$ and consider $r$ the line passing through the origin and $y_{0}$. Then, define $\Omega_{0}$ as the open connected region between $r$ and one of the axis, such that the angle between $r$ and the axis is $\leq \frac{\pi}{2}$. Taking $\delta>0$ such that $\delta<\left|y_{0}\right|$, set $\Omega=\Omega_{0} \cap B_{\delta}$. So, for $x \in \Omega$, we have that $\left|x-y_{0}\right|>\left|y_{0}\right|$. Therefore, by the Mean Value Theorem, there exists $x_{\delta} \in \Omega$, satisfying

$$
\begin{aligned}
\left\|u_{n}\right\|_{*}^{2} & \geq \int_{\Omega} \ln \left(1+\left|x-y_{n}\right|\right)\left|\tilde{u}_{n}(x)\right|^{p} d x \\
& =|\Omega| \ln \left(1+\left|x_{\delta}-y_{n}\right|\right)\left|\tilde{u}_{n}\left(x_{\delta}\right)\right|^{p} \\
& =C_{4} \ln \left(1+\left|x_{\delta}-y_{0}\right|\right) \geq C_{4} \ln \left(1+\left|y_{0}\right|\right)=C_{4} \ln \left(1+\left|y_{n}\right|\right),
\end{aligned}
$$

for $C_{4}>0$. If $y_{0}=0, \tilde{u}_{n}=u_{n}$ and the result follows immediately from (4.1). So, in any of the cases, there exists $C_{4}>0$ such that

$$
\left\|u_{n}\right\|_{*}^{p}=\int_{\mathbb{R}^{N}} \ln \left(1+\left|x-y_{n}\right|\right)\left|\tilde{u}_{n}(x)\right|^{p} d x \geq C_{4} \ln \left(1+\left|y_{n}\right|\right), \forall n \in \mathbb{N} .
$$


Now, we have

$$
\left\|\tilde{u}_{n}\right\|_{*}^{p}=\int_{\mathbb{R}^{N}} \ln \left(1+\left|x+y_{n}\right|\right)\left|u_{n}(x)\right|^{p} d x \leq\left\|u_{n}\right\|_{*}^{p}+\ln \left(1+\left|y_{n}\right|\right)\left\|u_{n}\right\|_{p}^{p} .
$$

From this, since every norm is weakly lower semicontinuos, $\|\cdot\| \|_{p}$ is $\mathbb{Z}^{N}$-invariant and $\tilde{u}_{n} \rightarrow u$ in $X$, follows that

$$
\begin{aligned}
\left\|\left(-y_{n}\right) * u\right\|_{*}^{p} & =\int_{\mathbb{R}^{N}} \ln \left(1+\left|x-y_{n}\right|\right)|u(x)|^{p} d x \\
& \leq\left\|\tilde{u}_{n}\right\|_{X}^{p}+\ln \left(1+\left|y_{n}\right|\right)\left\|u_{n}\right\|_{p}^{p} \\
& \leq\left\|u_{n}\right\|^{p}+\left\|u_{n}\right\|_{*}^{p}\left(1+C_{5}\left\|u_{n}\right\|_{p}^{p}\right) \\
& \leq\left\|u_{n}\right\|^{p}+C_{6}\left\|u_{n}\right\|_{*}^{p} \leq C_{7}\left\|u_{n}\right\|_{X}^{p}
\end{aligned}
$$

for $n \in \mathbb{N}$ and $C_{7}>0$. Consequently, there exists a constant $C_{8}>0$ such that, after passing to a subsequence, we have, for all $n \in \mathbb{N}$,

$$
\left\|\left(-y_{n}\right) * u\right\|_{X}^{p}=\|u\|^{p}+\left\|\left(-y_{n}\right) * u\right\|_{*}^{p} \leq\left\|u_{n}\right\|^{p}+C_{7}\left\|u_{n}\right\|_{X}^{p} \leq C_{8}\left\|u_{n}\right\|_{X}^{p} .
$$

Therefore, from (4.1) and (4.2),

$$
\left|I^{\prime}\left(\tilde{u}_{n}\right)\left(\tilde{u}_{n}-u\right)\right| \leq\left(1+C_{8}^{\frac{1}{p}}\right)|| I^{\prime}\left(u_{n}\right)\left\|_{X^{\prime}}|| u_{n}\right\|_{X} \rightarrow 0,
$$

finishing the claim.

Claim 3: $\int_{\mathbb{R}^{N}} f\left(\tilde{u}_{n}\right)\left(\tilde{u}_{n}-u\right) d x \rightarrow 0$, as $n \rightarrow+\infty$.

Since $\|\cdot\|$ is $\mathbb{Z}^{N}$-invariant, Lemma 2.2, Lemma 3.3 and Remark 3.1 remains valid for $\left(\tilde{u}_{n}\right)$. Moreover, $\frac{1}{2 p}+\frac{2 p-1}{2 p}=1, \frac{2 p}{2 p-1}(q-1)>p$ and $\omega_{0}=\frac{2 p}{2 p-1}(q-1) t_{0}>p$. Thus, from the boundedness of $\left(\tilde{u}_{n}\right)$ in $L^{\omega_{0}}\left(\mathbb{R}^{N}\right)$ and in $L^{p}\left(\mathbb{R}^{N}\right)$, Remark 3.1, Proposition 2.1, (2.5) and Hölder inequanlity, we have

$$
\begin{aligned}
\left|\int_{\mathbb{R}^{N}} f\left(\tilde{u}_{n}\right)\left(\tilde{u}_{n}-u\right) d x\right| & \leq \int_{\mathbb{R}^{N}}\left|\tilde{u}_{n}\right|^{p-1}\left|\tilde{u}_{n}-u\right| d x+b_{1} \int_{\mathbb{R}^{N}}\left|\tilde{u}_{n}\right|^{q-1}\left|\tilde{u}_{n}-u\right| R\left(\alpha, \tilde{u}_{n}\right) d x \\
& \leq C\left\|\tilde{u}_{n}-u\right\|_{p}+b_{1}\left\|\tilde{u}_{n}-u\right\|_{2 p}\left(\int_{\mathbb{R}^{N}} R\left(\alpha, \tilde{u}_{n}\right)^{\frac{2 p}{2 p-1}}\left|\tilde{u}_{n}\right|^{\frac{2 p}{2 p-1}(q-1)} d x\right)^{\frac{2 p-1}{2 p}} \\
& \leq C\left\|\tilde{u}_{n}-u\right\|_{p}+b_{1} K_{1}\left\|\tilde{u}_{n}-u\right\|_{2 p}|| \tilde{u}_{n} \|_{\omega_{0}}^{q-1} \rightarrow 0,
\end{aligned}
$$

as $n \rightarrow+\infty$, proving the claim.

Moreover, we observe that

(i) From HLS, Hölder inequality and Proposition 2.1,

$$
\left|V_{2}^{\prime}\left(\tilde{u}_{n}\right)\left(\tilde{u}_{n}-u\right)\right| \leq K_{0}\left\|\tilde{u}_{n}\right\|_{\frac{2 N}{2 N-1} p}^{2 p-1}\left\|\tilde{u}_{n}-u\right\|_{\frac{2 N}{2 N-1} p} \rightarrow 0 .
$$


(ii) $\left.\left|\int_{\mathbb{R}^{N}}\right| \tilde{u}_{n}\right|^{p-2} \tilde{u}_{n}\left(\tilde{u}_{n}-u\right) d x\left|\leq\left\|\tilde{u}_{n}\right\|_{p}^{p-1}\right| \mid \tilde{u}_{n}-u \|_{p} \rightarrow 0$.

(iii) We recall a standard result: there exists a constant $D_{1}>0$, depending only on $p$, such that

$$
|a-b|^{p} \leq D_{1}\left(|a|^{p-2} a-|b|^{p-2} b\right)(a-b), \forall a, b \in \mathbb{R}, \forall p \geq 2 .
$$

So,

$$
\begin{aligned}
V_{1}^{\prime}\left(\tilde{u}_{n}\right)\left(\tilde{u}_{n}-u\right) & =\int_{\mathbb{R}^{N}} \int_{\mathbb{R}^{N}} \ln (1+|x-y|)\left|\tilde{u}_{n}(x)\right|^{p}\left|\tilde{u}_{n}(y)\right|^{p-2} \tilde{u}_{n}(y)\left(\tilde{u}_{n}(y)-u(y)\right) d x d y \\
& \geq C_{9} \int_{\mathbb{R}^{N}} \int_{\mathbb{R}^{N}} \ln (1+|x-y|)\left|\tilde{u}_{n}(x)\right|^{p}\left|\tilde{u}_{n}(y)-u(y)\right|^{p} d x d y \\
& +\int_{\mathbb{R}^{N}} \int_{\mathbb{R}^{N}} \ln (1+|x-y|)\left|\tilde{u}_{n}(x)\right|^{p}|u(y)|^{p-2} u(y)\left(\tilde{u}_{n}(y)-u(y)\right) d x d y \\
& =C_{9} A_{1}+B_{1} .
\end{aligned}
$$

Note that $A_{1} \geq 0$ and, from Lemma 3.1, $B_{1} \rightarrow 0$, as $n \rightarrow+\infty$. Hence, $V_{1}^{\prime}\left(\tilde{u}_{n}\right)\left(\tilde{u}_{n}-u\right) \rightarrow 0$.

Hence, from Claims 2 and 3 and items (i)-(iii), we conclude that

$$
\begin{aligned}
o(1)=I^{\prime}\left(\tilde{u}_{n}\right)\left(\tilde{u}_{n}-u\right) & =\tilde{A}\left(\tilde{u}_{n}\right)\left(\tilde{u}_{n}-u\right)+V_{0}^{\prime}\left(\tilde{u}_{n}\left(\tilde{u}_{n}-u\right)-\int_{\mathbb{R}^{N}} f\left(\tilde{u}_{n}\right)\left(\tilde{u}_{n}-u\right) d x\right. \\
& \geq \tilde{A}\left(\tilde{u}_{n}\right)\left(\tilde{u}_{n}-u\right)+o(1) .
\end{aligned}
$$

That is, $\tilde{A}\left(\tilde{u}_{n}\right)\left(\tilde{u}_{n}-u\right) \rightarrow 0$. So, once $\tilde{A}$ has the $(S)$ property, $\tilde{u}_{n} \rightarrow u$ in $W^{s, p}\left(\mathbb{R}^{N}\right)$. Therefore, we can also conclude that $A_{1} \rightarrow 0$ and, from Proposition 3.1, $\left\|\tilde{u}_{n}-u\right\|_{*} \rightarrow 0$, proving that $\tilde{u}_{n} \rightarrow u$ in $X$.

Finally, remains to show that $u$ is a critical point of $I$. Let $v \in X$. So, as we did above, is possible to find $C_{10}>0$ such that $\left\|\left(-y_{n}\right) * v\right\|_{X} \leq C_{10}\left\|u_{n}\right\|_{X}$. Thus,

$$
\left|I^{\prime}(u)(v)\right|=\lim \left|I^{\prime}\left(\tilde{u}_{n}\right)(v)\right|=\lim \left|I^{\prime}\left(u_{n}\right)\left(\left(-y_{n}\right) * v\right)\right| \leq C_{10} \lim || I^{\prime}\left(u_{n}\right)\left\|_{X^{\prime}}|| u_{n}\right\|_{X}=0 .
$$

Therefore, $u$ is a nontrivial critical point for $I$ in $X$.

Proof of Theorem 1.1. (i) From Lemma 3.4 and Proposition 4.1 there exists a nontrivial critical point of $I, u_{0} \in X$, such that $I\left(u_{0}\right)=c_{m p}$.

(ii) We start defining the set $\mathcal{K}=\left\{v \in X \backslash\{0\} ; I^{\prime}(v)=0\right\}$. Since $u_{0} \in \mathcal{K}, \mathcal{K} \neq \emptyset$. Thus, we can consider a sequence $\left(u_{n}\right) \subset \mathcal{K}$ satisfying $I\left(u_{n}\right) \rightarrow c_{g}=\inf _{v \in \mathcal{K}} I^{\prime}(v)$.

Observe that $c_{g} \in\left[-\infty, c_{m p}\right]$. If $c_{q}=c_{m p}$ the proof is finished. Otherwise, if $c_{g}<c_{m p}$, considering a subsequence if necessary, we can suppose that $I\left(u_{n}\right) \leq c_{m p}$, for all $n \in \mathbb{N}$ and, from the definition of $\mathcal{K}$, we see that $\left(u_{n}\right)$ satisfies $\left\|I^{\prime}\left(u_{n}\right)\right\|_{X^{\prime}}\left(1+\left\|u_{n}\right\|_{X}\right) \rightarrow 0$. Moreover, since $I^{\prime}\left(u_{n}\right)\left(u_{n}\right)=0$, for all $n \in \mathbb{N}$, from (3.5), $\left\|u_{n}\right\|>\rho$, for all $n \in \mathbb{N}$. Therefore, from Proposition 4.1 there exists $\left(y_{n}\right) \subset \mathbb{Z}^{N}$ such that $\tilde{u}_{n} \rightarrow u$ in $X$, for a nontrivial critical point $u$ 
of $I$ in $X$. Consequently, $I^{\prime}(u)=\lim I^{\prime}\left(\tilde{u}_{n}\right)=\lim I^{\prime}\left(u_{n}\right)=0$ and we conclude that $u \in \mathcal{K}$ and

$$
I(u)=\lim I\left(\tilde{u}_{n}\right)=\lim I\left(u_{n}\right)=c_{g} .
$$

Particularly, $c_{g}>-\infty$.

\section{Proof of Theorem 1.2}

In this section we will provide the proof of the multiplicity result stated in Theorem 1.2. In order to do so, we will need to verify some results concerned with the genus theory, denoted by $\gamma$ and whose definition, given over $\mathcal{A}=\{A \subset X ; A$ is symmetric and closed $\}$ (with respect to continuity in $X$ ), and basic properties can be found in [33] Chapter II.5. We start this section, verifying some properties of an important auxiliary function, namely, $\varphi_{u}: \mathbb{R} \rightarrow \mathbb{R}$, given by $\varphi_{u}(t)=I(t u)$, for all $u \in X \backslash\{0\}$ and $t \in \mathbb{R}$.

Once the results are done similarly as in [5], in order to make the paper concise, we will only sketch the proofs here (see also [10]).

Lemma 5.1. (a) Let $u \in X \backslash\{0\}$. Then, $\varphi_{u}$ is even and there exists a unique $t_{u} \in(0,+\infty)$ such that $\varphi_{u}^{\prime}(t)>0$, for all $t \in\left(0, t_{u}\right)$, and $\varphi_{u}^{\prime}(t)<0$, for all $t \in\left(t_{u}, \infty\right)$. Moreover, $\varphi_{u}(t) \rightarrow-\infty$, as $t \rightarrow+\infty$.

(b) Let $u \in X \backslash\{0\}$. Then, there exists a unique $t_{u}^{\prime} \in(0,+\infty)$ such that $\varphi_{u}(t)>0$, for $t \in\left(0, t_{u}^{\prime}\right)$, and $\varphi_{u}(t)<0$, for $t \in\left(t_{u}^{\prime},+\infty\right)$. Moreover, $t_{u}$ given by item (a) is a global maximum for $\varphi_{u}$.

(c) For each $u \in X \backslash\{0\}$, the map $u \mapsto t_{u}^{\prime}$ is continuous.

Proof. We prove item (a). Since $f$ is odd, $I$ is even and, consequently, $\varphi_{u}$ is even as well.

(i) For $t>0$ sufficiently small and $\alpha>0$, for (2.5), we have

$$
\varphi_{u}^{\prime}(t) \geq t^{p-1}\|u\|^{p}\left[1-C_{2} t^{2(p-1)}\|u\|^{p}-\varepsilon-C_{4} t^{q-p}\|u\|^{q-p}\right] .
$$

Thus, $\varphi_{u}^{\prime}(t)>0$ for $t, \varepsilon>0$ sufficiently small.

(ii) From $\left(f_{3}\right)$ and $\left(f_{4}^{\prime}\right)$, once $q>2 p$,

$$
\varphi_{u}^{\prime}(t) \leq t^{p-1}\|u\|^{p}+t^{2 p-1} V_{1}(u)-C_{3} t^{q-1}\|u\|_{q}^{q} \rightarrow-\infty, \text { as } t \rightarrow+\infty .
$$

Hence, from (i)-(ii), since $I$ is $C^{1}$, there exists $t_{u} \in(0,+\infty)$ such that $\varphi_{u}^{\prime}\left(t_{u}\right)=0$, which is unique by $\left(f_{5}\right)$.

Item (b) follows as a consequence of item (a) and item (c) as a consequence of predecessors.

Now, we define the following sets

$$
K_{c}=\left\{u \in X ; I^{\prime}(u)=0, I(u)=c\right\}, c \in(0,+\infty)
$$

and

$$
A_{c, \rho}\left\{u \in X ;\|u-v\| \leq \rho \text {, for some } v \in K_{c}\right\}, c \in(0,+\infty)
$$


It is easy to verify that the sets $K_{c}$ and $A_{c, \rho}$ are symmetric, closed (with respect to $X$ ) and invariant under $\mathbb{Z}^{N}$ translations, i.e, if $u \in K_{c}, A_{c, \rho}$, then $z * \in K_{c}, A_{c, \rho}$, for all $z \in \mathbb{Z}^{N}$.

Next, we fix a continuous map $\beta: L^{p}\left(\mathbb{R}^{N}\right) \backslash\{0\} \rightarrow \mathbb{R}^{N}$ that is equivariant under $\mathbb{Z}^{N}$ translations, that is, $\beta(x * u)=x+\beta(u)$, for $x \in \mathbb{Z}^{N}$ and $u \in L^{p}\left(\mathbb{R}^{N}\right) \backslash\{0\}$. We also require that $\beta(-u)=\beta(u)$. Such map is called a generalized barycenter map and an example can be constructed as in [4]. Hence, we can define

$$
\tilde{K}_{c}=\left\{u \in K_{c} ; \beta(u) \in[-4,4]^{N}\right\},
$$

which are clearly symmetric sets. Moreover, before given our first result, we need to recall the Gauss bracket $[\cdot]: \mathbb{R} \rightarrow \mathbb{Z}$, given by $[s]=\max \{n \in \mathbb{Z} ; n \leq s\}$ (see [19, Chapter 3]), which naturally induce a map from $\mathbb{R}^{N}$ onto $\mathbb{Z}^{N}$, as follows

$$
\left[\left(x_{1}, x_{2}, \ldots, x_{N}\right)\right]=\left(\left[x_{1}\right],\left[x_{2}\right],\left[x_{N}\right]\right) \in \mathbb{Z}^{N}, \forall\left(x_{1}, x_{2}, \ldots, x_{N}\right) \in \mathbb{R}^{N}
$$

We recall some properties of the Gauss bracket, that are needed inside of the proof of our results.

Lemma 5.2. Let $[\cdot]: \mathbb{R} \rightarrow \mathbb{Z}$, given by $[s]=\max \{n \in \mathbb{Z} ; n \leq s\}$. Then,

(i) $0 \leq s-[s]<1$, for all $s \in \mathbb{R}$.

(ii) if $z \in \mathbb{Z}$ and $s \in \mathbb{R}$, then $[z+s]=z+[s]$.

(iii) Let $s \in \mathbb{R}$ such that $s-[s] \geq \frac{1}{2}$, then $s-\frac{1}{2}-\left[s-\frac{1}{2}\right]<\frac{1}{2}$.

(iv) $0 \leq s-[s]<1$, for all $s \in \mathbb{R}$.

(v) Let $s \in \mathbb{R}$. Then, $s-\left[s-\frac{1}{2}\right]<1$.

Proposition 5.1. Let $c>0$. Then, there exists $\rho_{0}=\rho_{0}(c)>0$ such that $\gamma\left(A_{c, \rho}\right)<\infty$, for all $\rho \in\left(0, \rho_{0}\right)$.

Proof. The proof of this proposition can be done similarly as [5, Proposition 4.1], with minor changes. We only highlight how to construct the sets $L_{i}$ inside the refereed proof, once here we need $2^{N}$ sets to "cover" $L^{p}\left(\mathbb{R}^{N}\right) \backslash\{0\}$.

Let $\left\{e_{i}\right\}_{i=1}^{N}$ be the canonical base of $\mathbb{R}^{N}$. Consider $a_{i}=\frac{1}{2} e_{i}, a_{i j}=\frac{1}{2} e_{i}+\frac{1}{2} e_{j}, a_{i j k}=\frac{1}{2}\left(e_{i}+\right.$ $\left.e_{j}+e_{k}\right)$, for $1 \leq i, j, k \leq N$, and successively until $a_{12 \cdots N}=\frac{1}{2} \sum_{i=1}^{N} e_{i}$. Observe that $a_{12}=a_{21}$, so, excluding the repeating cases, we define $2^{N}-1$ sets as $L_{i}=a_{i} * L_{1} \subset L^{p}\left(\mathbb{R}^{N}\right) \backslash\{0\}$, $L_{i j}=a_{i j} * L_{1} \subset L^{p}\left(\mathbb{R}^{N}\right) \backslash\{0\}, \ldots, L_{12 \cdots N}=a_{12 \cdots N} * L_{1} \subset L^{p}\left(\mathbb{R}^{N}\right) \backslash\{0\}$. Once this construction is well understood, in order to simplify the notation, we simply denote $a_{i}$ and $L_{i}$, for $2 \leq i \leq 2^{N}$.

It is clear that $L^{p}\left(\mathbb{R}^{N}\right) \backslash\{0\} \subset \bigcup_{i=1}^{2^{N}} L_{i}$.

For the next results we will need the definition and some basic properties of relative genus. So, for convenience of the reader, we will include it here.

Definition 5.1. Let $D, Y \in \mathcal{A}$ with $D \subset Y$. We say that $U, V \in \mathcal{A}$ is a covering of $Y$ relative to $D$ if is satisfies

(i) $Y \subset U \cup V$ and $D \subset U$; 
(ii) there exists an even continuous (in $X)$ function $\chi: U \rightarrow D$, such that $\chi(u)=u$, for all $u \in D$.

If $U, V \in \mathcal{A}$ is a covering of $Y$ relative to $D$, then the genus of this covering is $\gamma(V)=k$.

Definition 5.2. Let $D, Y \in \mathcal{A}$ with $D \subset Y$. We define the Krasnoselskii's Genus of $Y$ relative to $D$, denoted by $\gamma_{D}(Y)$, as

(i) There exists a covering for $Y$ relative to $D$ and, in this case, $\gamma_{D}(Y)=k$, where $k$ is the lowest genus of this coverings.

(ii) If we cannot find any such covering of $Y$ relative to $D$, we set $\gamma_{D}(Y)=+\infty$.

In the following, we list some useful properties of relative genus that are needed to guarantee that the results are valid.

Lemma 5.3. (i) Let $D \subset \mathcal{A}$. Then, $\gamma_{D}(D)=0$.

(ii) Let $D, Y, Z \in \mathcal{A}$ satisfying $D \subset Y$ and $D \subset Z$. If there exists a function $\varphi: Y \rightarrow Z$, even and continuous (in $X$ ), such that $\varphi(u)=u$, for all $u \in D$, then $\gamma_{D}(Y) \leq \gamma_{D}(Z)$.

(iii) Let $D \subset Y \subset Z \in \mathcal{A}$. Then, $\gamma_{D}(Y) \leq \gamma_{D}(Z)$.

(iv) Let $D, Y, Z \in \mathcal{A}$ satisfying $D \subset Y$. Then, $\gamma_{D}(Y \cup Z) \leq \gamma_{D}(Y)+\gamma(Z)$.

Proof. For item (i), take $U=D, V=\emptyset$ and $\chi=i d$, in the definition of relative genus. Proofs for itens (ii) and (iv) can be found, for example, in [10]. Finally, item (iii) is an immediate consequence of item (ii).

For the next results, we define the sets

$$
I^{c}=\{u \in X ; I(u) \leq c\}, \text { for } c \in \mathbb{R} \text { and } D=I^{0}
$$

and the values

$$
c_{k}=\inf \left\{c \geq 0 ; \gamma_{D}\left(I^{c}\right) \geq k\right\}, \forall n \in \mathbb{N} .
$$

Remark 5.1. (1) Since $I$ is unbounded from bellow, $D \neq \emptyset$.

(2) Let $c_{1}, c_{2} \in \mathbb{R}$ with $c_{1}>c_{2}$. Then, if $u \in I^{c_{2}}, I(u) \leq c_{2}<c_{1}$, so $u \in I^{c_{2}}$. That is, if $c_{1}>c_{2}$, then $I^{c_{2}} \subset I^{c_{1}}$.

(3) If $c_{1}>c_{2} \geq 0$, then $D \subset I^{c_{2}} \subset I^{c_{1}}$. Consequently, $\gamma_{D}\left(I^{c_{2}}\right) \leq \gamma_{D}\left(I^{c_{1}}\right)$.

(4) For $\varepsilon>0, \gamma_{D}\left(I^{c_{k}+\varepsilon}\right) \geq k$ and $\gamma_{D}\left(I^{c_{k}-\varepsilon}\right)<k$, for every $k \in \mathbb{N}$.

(5) $\inf _{u \in X \backslash\{0\}} \sup _{t \in \mathbb{R}} I(t u)=\inf _{u \in X \backslash\{0\}} \sup _{t>0} I(t u)<+\infty$.

Consider the Nehari's manifold for $I$, defined by

$$
\mathcal{N}=\left\{u \in X \backslash\{0\} ; I^{\prime}(u)(u)=0\right\}
$$

Lemma 5.4. Let $\mathcal{N}$ as in (5.1). Then,

$$
\inf _{\mathcal{N}} I=\inf _{u \in X \backslash\{0\}} \sup _{t>0} I(t u)
$$

Proof. From Lemma 5.1 and the chain rule, for $u \in X \backslash\{0\}$, we have $t_{u} u \in \mathcal{N}$ and $\sup _{t>0} I(t u)=$ $I\left(t_{u} u\right)$. Moreover, for $u \in \mathcal{N}$, once again from Lemma 5.1, $\sup _{t>0} I(t u)=I(u)$. Therefore, the result follows. 
Lemma 5.5. We have $\inf _{X \backslash\{0\}} \sup _{t \in \mathbb{R}} I(t u)>0$.

Proof. From Lemmas 5.4 and 3.4 and direct calculations, one can obtain the result.

In order to prove the next results, we need to introduce the following sets

$$
\mathcal{N}^{+}=\left\{u \in X ; I^{\prime}(u)(u)>0\right\} \text { and } \mathcal{N}^{-}=\left\{u \in X ; I^{\prime}(u)(u)<0\right\} .
$$

Note that $X=\{0\} \cup \mathcal{N}^{+} \cup \mathcal{N} \cup \mathcal{N}^{-}$. Moreover, using the definition of a set's boundary and Lemmas 3.4 and 5.1, it is possible to verify that $\partial \mathcal{N}^{-}=\mathcal{N}$ and $\partial \mathcal{N}^{+}=\{0\} \cup \mathcal{N}$.

Proposition 5.2. We have $c_{1}=\inf _{N} I=\inf _{u \in X \backslash\{0\}} \sup _{t>0} I(t u)>0$.

Proof. Observe that, from Lemma 5.4, remains to prove that $c_{1}=\inf _{N} I>0$. From Lemma 5.3-(i), $c_{1}>0$.

Claim 1: $c_{1} \geq \inf _{\mathcal{N}} I$.

Suppose, by contradiction, that $c_{1}<\inf _{\mathcal{N}} I$. Choose $c \in\left(c_{1}, \inf _{\mathcal{N}} I\right)$. Define the function $F: I^{c} \rightarrow X$ by

$$
F(u)=\left\{\begin{array}{l}
0, \text { if } u \in\{0\} \cup \mathcal{N}^{+} \\
\max \left\{1, t_{u}^{\prime}\right\} u, \text { if } u \in \mathcal{N}^{-}
\end{array} .\right.
$$

We see that $F$ is well-defined, continuous, odd and $\left.F\right|_{D}=i d$ Consider $U=I^{c}$ and $V=$ $\emptyset$. Since $I$ is $C^{1}$ and odd, $U$ is closed and symmetric. Hence, $\gamma_{D}\left(I^{c}\right)=0$. But it gives a contradiction, since $1 \leq \gamma_{D}\left(I^{c_{1}}\right) \leq \gamma_{D}\left(I^{c}\right)=0$. Consequently, $c_{1} \geq \inf _{\mathcal{N}} I$.

Claim 2: $c_{1} \leq \inf _{\mathcal{N}} I$.

For $u_{0} \in X \backslash\{0\}$, without loss of generality, we can assume $\left\|u_{0}\right\|=1$. Set $d=\sup _{t>0} I\left(t u_{0}\right)$. Lets prove that $\gamma_{D}\left(I^{d}\right) \geq 1$. Note that, if $u \in B=\left\{t u_{0} ; t>0\right\}$, then there exists $t_{0}>0$ such that $u=t_{0} u_{0}$. One can see that $D \subset B \cup D \subset I^{d}$.

Thus, from Lemma 5.3-(iii), $\gamma_{D}(B \cup D) \leq \gamma_{D}\left(I^{d}\right)$. In this point of view, we work to prove that $\gamma_{D}(B \cup D) \geq 1$.

Suppose that $\gamma_{D}(B \cup D)=0$. Then, by definition, $U=B \cup D$ and $V=\emptyset$, once only the empty set has null genus. Moreover, there exists a function, continuous and odd, $\chi: B \cup D \rightarrow D$ such that $\chi(u)=u$, for all $u \in D$.

Define $g:(0,+\infty) \rightarrow(0,+\infty)$ by $g(t)=\left\|\chi\left(t u_{0}\right)\right\|$. Note that $g$ is continuous. Hence, from Lemmas 3.4 and 5.1 and the Intermediate Value Theorem, we reach a contradiction. Therefore, claim 2 is valid and, combined with claim 1, we have the proposition.

Remark 5.2. (1) We can provide an equivalent definition to the the function $F: I^{c} \rightarrow X$ by

$$
F(u)=\left\{\begin{array}{l}
0, \text { if } u \in\{0\} \cup \mathcal{N}^{+} \\
\sigma(u) u, \text { if } u \in \mathcal{N}^{-}
\end{array},\right.
$$

where $\sigma: \mathcal{N}^{-} \rightarrow[1,+\infty)$ is given by $\sigma(u)=\inf \left\{t \geq 1 ; \varphi_{u}(t)=I(t u) \leq 0\right\}$.

(2) Let $i, j \in \mathbb{N}$ with $i>j$. Then,

$$
\left\{c \in(0,+\infty) ; \gamma_{D}\left(I^{c}\right) \geq i\right\} \subset\left\{c \in(0,+\infty) ; \gamma_{D}\left(I^{c}\right) \geq j\right\}
$$


Therefore, $c_{j} \leq c_{i}$.

In the following, we will consider $W$ as a $k$-dimensional subspace of $X$. As a consequence of $\left(f_{1}^{\prime}\right),\left(f_{4}^{\prime}\right), V_{2}(u) \geq 0$ and $V_{1}(u) \leq 2\|u\|_{X}^{2 p}$, once can see that

$$
I(u) \rightarrow-\infty, \text { as }\|u\|_{X} \rightarrow+\infty, \text { and } \sup _{u \in W} I(u)<+\infty
$$

Corolary 5.1. There exists $R>0$ such that $\{u \in W ;\|u\| \geq R\} \subset D$.

For the next results, consider $\rho>0$ given by Lemma 3.4 and $\chi: X \rightarrow X$ a continuous and even function, such that $\chi(u)=u$, for all $u \in D$. Define the sets

$$
\mathcal{O}_{\chi}=\{u \in W ;\|\chi(u)\|<\rho\}
$$

For convenience, we set $\chi$ as a function having these properties, except we say otherwise.

Remark 5.3. One can easily verify that the sets $\mathcal{O}_{\chi}$ have the following properties:

(1) $\mathcal{O}_{\chi}$ is a neighbourhood of zero in $W$.

(2) $\mathcal{O}_{\chi}$ is bounded and symmetric.

(3) If $u \in \partial_{W} \mathcal{O}_{\chi}$, then $\|\chi(u)\|=\rho$.

Proposition 5.3. Let $k \in \mathbb{N}$. Then $c_{k}<+\infty$.

Proof. Set $\alpha=\sup _{u \in W} I(u)$. From (5.2), $\alpha<+\infty$. Also, by definition of $I^{\alpha}$, we see that $W \subset I^{\alpha}$.

Suppose that $\gamma_{D}\left(I^{\alpha}\right)<k$. Then, from Definition 5.2, a corollary of Tietze's Theorem, Remark 5.3 and a genus property, $\gamma\left(\partial_{W} \mathcal{O}_{\chi}\right)=k$.

Let $u \in \partial_{W} \mathcal{O}_{\chi}$. From Remark 5.3, $\|\chi(u)\|=\rho$. By Lemma 3.4, $I(\chi(u)) \geq m_{\rho}>0$. Thus, $\chi(u) \notin D$ and $\partial_{W} \mathcal{O}_{\chi} \cap U=\emptyset$.

On the other hand,

$$
\partial_{W} \mathcal{O}_{\chi} \subset W \subset I^{\alpha} \subset U \cup V \Longrightarrow \partial_{W} \mathcal{O}_{\chi} \subset V
$$

Then, we have $k=\gamma\left(\partial_{W} \mathcal{O}_{\chi}\right) \leq \gamma(V) \leq k-1$, which is a contradiction.

Therefore, from the definition of values $c_{k}$ and Lemma 5.2, $c_{k} \leq \alpha<+\infty$.

Before we prove that the values $c_{k}$ are critical values of $I$, we will provide a deformation lemma. We start defining the sets $S=X \backslash A_{c, \rho}$,

$$
S_{\delta}=\left\{u \in X ;\|u-v\|_{X} \leq \delta, \text { for some } v \in S\right\}
$$

and

$$
\tilde{S}_{\delta}=\{u \in X ;\|u-v\| \leq \delta, \text { for some } v \in S\},
$$

for $c, \rho, \delta \in(0,+\infty)$. Since the proofs of Lemma 5.6 and Corollary 5.2 can be done as [10, Lemma 4.6], we omit it here.

Lemma 5.6. Let $c, \rho>0$. Then, there exists $\delta_{0}=\delta(c, \rho)>0$ such that, if $\delta \in\left(0, \delta_{0}\right)$, then $\left\|I^{\prime}(u)\right\|_{X^{\prime}}\left(1+\|u\|_{X}\right) \geq 8 \delta$, for all $u \in \tilde{S}_{2 \delta}$ with $I(u) \in\left[c-2 \delta^{2}, c+2 \delta^{2}\right]$. 
Corolary 5.2. Let $c, \rho>0$. Then, there exists $\tilde{\delta}_{0}=\tilde{\delta}_{0}(c, \rho)>0$ such that, if $\delta \in\left(0, \tilde{\delta}_{0}\right)$, then $\left\|I^{\prime}(u)\right\|_{X^{\prime}}\left(1+\|u\|_{X}\right) \geq 8 \delta$, for all $u \in S_{2 \delta}$ with $I(u) \in\left[c-2 \delta^{2}, c+2 \delta^{2}\right]$.

Lemma 5.7. Let $c>0$. Then, there exists $\rho_{1}=\rho_{1}(c)>0$ such that, for all $\rho \in\left(0, \rho_{1}\right)$, we have (i) $A_{c, \rho} \cap D=\emptyset$.

(ii) There exists $\varepsilon=\varepsilon(c, \rho)>0$ and a function $\phi: I^{c+\varepsilon} \backslash A_{c, \rho} \rightarrow I^{c-\varepsilon}$, continuous and even, such that $D \subset I^{c-\varepsilon}$ and $\left.\phi\right|_{D}=i d$.

Proof. Item (i) follows from the application of Proposition 4.1 and Lemma 3.4, arguing by contraction. For item (ii), let $\delta_{0}$ as given by Lemma 5.2 and $\delta \in\left(0, \delta_{0}\right)$, such that $\delta^{2}<\frac{c}{2}$. Take $\varepsilon=\delta^{2}$. Then, from Deformation's Lemma 2.6 of [26], there exists $\eta:[0,1] \times X \rightarrow X$, continuous, satisfying

(a) $\eta(t, u)=u$, if $t=0$ or $u \notin I^{-1}([c-2 \varepsilon, c+2 \varepsilon]) \cap S_{2 \delta}$;

(b) $\eta\left(1, I^{c+\varepsilon} \cap\left(X \backslash A_{c, \rho}\right)\right) \subset I^{c-\varepsilon}$;

(c) $t \mapsto I(\eta(t, u))$ is non-increasing, for all $u \in X$.

Moreover, since $I$ is odd, it is possible to modify the proof of the refereed lemma, such as in [?], to obtain as well

(d) $\eta(t,-u)=-\eta(t, u)$, for all $t \in[0,1]$ and $u \in X$.

Define $\phi: I^{c+\varepsilon} \backslash A_{c, \rho} \rightarrow I^{c-\varepsilon}$ by $\phi(u)=\eta(1, u)$. Note that item (b) is equivalent to $\eta\left(1, I^{c+\varepsilon} \cap \backslash A_{c, \rho}\right) \subset I^{c-\varepsilon}$, which guarantee that $\phi$ is well-defined. Also, as $\eta$ is continuous, $\phi$ is continuous as well, and from item (d), $\phi$ is even.

Moreover, once $\varepsilon=\delta^{2}<\frac{c}{2}, 0 \notin[c-2 \varepsilon, c+2 \varepsilon]$. Thus, $D \cap I^{-1}([c-2 \varepsilon, c+2 \varepsilon])=\emptyset$. Hence, from item (a), if $u \in D, \phi(u)=\eta(1, u)=u$, which implies that $\left.\phi\right|_{D}=i d$.

Finally, if $u \in D, I(u) \leq 0<c-\varepsilon$, then $u \in I^{c-\varepsilon}$.

Proposition 5.4. Let $k \in \mathbb{N}$. Then, $c_{k}$ is a critical value of $I$.

Proof. Arguing by contradiction, from Lemma 5.7 and from items (ii) and (iii) of Lemma 5.3, one get that $c_{k}$ is a critical value of $I$.

Proposition 5.5. We have $c_{k} \rightarrow+\infty$, as $k \rightarrow+\infty$.

Proof. Suppose that there exists $M>0$, such that $c_{k}<M$, for all $k \in \mathbb{N}$. From Remark 5.2, $c_{k}$ is monotonically nondecreasing. Then, there exists $c>0$ such that $c_{k} \rightarrow c$.

From Proposition 5.1, there exists $\rho_{0}>0$ such that $\gamma\left(A_{c, \rho}\right)<+\infty$, for all $\rho \in\left(0, \rho_{0}\right)$. Also, from items (i) and (ii) of Lemma 5.7 and items (ii) and (iv) of Lemma 5.3, there exists $\varepsilon>0$ such that

$$
\gamma_{D}\left(I^{c+\varepsilon}\right)=\gamma_{D}\left(\left(I^{c+\varepsilon} \backslash A_{c, \rho}\right) \cup A_{c, \rho}\right) \leq \gamma_{D}\left(I^{c+\varepsilon} \backslash A_{c, \rho}\right)+\gamma\left(A_{c, \rho}\right)<+\infty
$$

Once more, as $c_{k} \rightarrow c$ monotonously nondecreasing and by definition of values $c_{k}$, we have $\gamma_{D}\left(I^{c+\varepsilon}\right) \rightarrow+\infty$, as $k \rightarrow+\infty$, which leads to a contradiction.

Therefore, $c_{k} \rightarrow+\infty$, as $k \rightarrow+\infty$.

Proof of Theorem 1.2. From Proposition 5.5, we can extract a subsequence of $\left(c_{k}\right)$ such that $c_{k} \rightarrow+\infty$ monotonously increasing. Then, from Proposition 5.4, there exists $u_{k} \in X$ satisfying $I\left(u_{k}\right)=c_{k}$ and $I^{\prime}\left(u_{k}\right)=0$, for all $k \in \mathbb{N}$. Since $\left(c_{k}\right)$ is monotone, $c_{i} \neq c_{j}$, when $i \neq j$, and $c_{k}>c_{1}>0$, for all $k \geq 2$. Then, we conclude that the functions $u_{k}$ are distinct and that $u_{k} \neq 0$, for all $n \in \mathbb{N}$. Also, as $I$ is odd, the same holds for $-u_{k}$ and $I\left( \pm u_{k}\right) \rightarrow+\infty$. 
Data availability statement: The data that supports the findings of this study are available within the article [and its supplementary material].

Acknowledgements: This work was perfomed and completed during the first author PhD graduate course at Federal University of São Carlos.

\section{References}

[1] Adams, R.A. and Fournier, J.J.F. (2014) Sobolev Spaces. Elsevier Science, Oxford.

[2] Alves, C.O. and Figueiredo, G.M. (2019) Existence of positive solution for a planar Schrödinger-Poisson system with exponential growth, Journal of Mathematical Physics. 60, 011503.

[3] Autuori, G., Pucci, P. (2013) Elliptic problems involving the fractional Laplacian in $\mathbb{R}^{N}$, Journal of Differential Equations. 255, 2340-2362.

[4] Bartsch T., Weth, T. Three nodal solutions of singularly perturbed elliptic equations on domains without topology, Annales de l'Institut Henri Poincare (C) Non Linear Analysis. 22 (2005) 259-281.

[5] Böer, E. de S. and Miyagaki, O.H. (2020) The Choquard logarithmic equation involving fractional Laplacian operator and a nonlinearity with exponential critical growth, Preprint in ArXiv:2011.12806v2 [Math].

[6] Bonheure, D., Cingolani, S., and Van Schaftingen, J. (2017) The logarithmic Choquard equation: Sharp asymptotics and nondegeneracy of the groundstate. Journal of Functional Analysis, 272, 5255-5281.

[7] Brézis, H. Functional analysis, Sobolev spaces and partial differential equations, Springer, New York; London, 2011.

[8] Cao, D. M. (1992) Nontrivial solution of semilinear elliptic equations with critical exponent in $\mathbb{R}^{2}$. Communications in Partial Differential Equations, 17, 407-435.

[9] Cingolani, S. and Jeanjean, L. (2019) Stationary waves with prescribed $L^{2}$-norm for the planar Schrödinger-Poisson system, SIAM J. Math. Anal. 51, 3533-3568.

[10] Cingolani, S. and Weth, T. (2016) On the planar Schrödinger-Poisson system. Annales de l'Institut Henri Poincare (C) Non Linear Analysis, 33, 169-197.

[11] Cui, N. and Sun, H. (2020) Fractional $p$-Laplacian problem with indefinite weight in $\mathbb{R}^{N}$ : Eigenvalues and existence, Math Meth Appl Sci., mma.6323.

[12] Demengel, F., Demengel, G. and Erné, R. (2012) Functional spaces for the theory of elliptic partial differential equations, Springer [u.a.], London.

[13] Di Nezza, E., Palatucci, G., Valdinoci E. (2012) Hitchhiker's guide to the fractional Sobolev spaces, Bulletin Des Sciences Mathématiques. 136 521-573. 
[14] do Ó, J. M., Medeiros, E. and Severo, U. (2008) A nonhomogeneous elliptic problem involving critical growth in dimension two, J. Math. Anal. Appl. 345, 286-304.

[15] do Ó, J.M., Miyagaki O.H. and Squassina, M. Ground states of nonlocal scalar field equations with Trudinger-Moser critical nonlinearity, ArXiv:1504.07291 [Math]. (2015).

[16] do Ó, J. M., Miyagaki, O. H., and Squassina, M. (2015) Nonautonomous fractional problems with exponential growth. Nonlinear Differential Equations and Applications No-DEA, $22,1395-1410$.

[17] Du, M. and Weth, T. (2017) Ground states and high energy solutions of the planar Schrödinger-Poisson system. Nonlinearity, 30, 3492-3515.

[18] Folland, G.B. Real analysis: modern techniques and their applications, 2nd ed, Wiley, New York, 1999.

[19] Graham, R.L., Knuth, D.E. and Patashnik, O. Concrete mathematics: a foundation for computer science, 2nd ed, Addison-Wesley, Reading, Mass, 1994.

[20] Guo, Q. and Wu, J.(2020) Existence of Solutions to the Logarithmic Choquard Equations in High Dimensions, Bull. Malays. Math. Sci. Soc. 43, 1545-1553.

[21] Iannizzotto, A. and Squassina, M. (2014) Weyl-type laws for fractional p-eigenvalue problems, ArXiv:1312.2441 [Math].

[22] Pucci, P., Xiang, M. and Zhang, B. (2015) Multiple solutions for nonhomogeneous Schrödinger-Kirchhoff type equations involving the fractional p-Laplacian in $\mathbb{R}^{N}$, Calc. Var. 54, 2785-2806.

[23] Pucci, P., Xiang, M. and Zhang, B. (2016) Existence and multiplicity of entire solutions for fractional $p$-Kirchhoff equations, Advances in Nonlinear Analysis. 5.

[24] Lam, N. and Lu, G. (2014) Elliptic equations and systems with subcritical and critical exponential growth without the Ambrosetti-Rabinowitz condition, J. Geom. Anal., 24,118143.

[25] Ledesma, C. E. T. (2017) Existence and symmetry result for fractional $p$-Laplacian in $\mathbb{R}^{N}$, Communications on Pure and Applied Analysis, 16, 99-113.

[26] Li, G. and Wang, C. The existence of a nontrivial solution to a nonlinear elliptic problem of linking type without the Ambrosetti-Rabinowitz condition, Ann. Acad. Sci. Fenn. Math. 36 (2011) 461-480.

[27] Li, Q. and Yang, Z. (2016) Multiple solutions for a class of fractional quasi-linear equations with critical exponential growth in $\mathbb{R}^{N}$, Complex Variables and Elliptic Equations. 61, 969-983.

[28] Lieb, E. H. (1977) Existence and uniqueness of the minimizing solution of Choquard's nonlinear equation. Studies in Applied Mathematics, 57, 93-105. 
[29] Lieb, E. H. (1983) Sharp constants in the Hardy-Littlewood-Sobolev and related inequalities. The Annals of Mathematics, 118, 349.

[30] Molica Bisci, G., Rădulescu, V.D. and Servadei, R. (2016) Variational methods for nonlocal fractional problems, Cambridge University Press, Cambridge.

[31] Moser, J. (1971) A Sharp form of an inequality by N. Trudinger. Indiana University Mathematics Journal, 20, 1077-1092.

[32] Perera, K., Agarwal, R.P. and O'Regan, D. Morse theoretic aspects of p-Laplacian type operators, American Mathematical Society, Providence, R.I, 2010.

[33] Struwe, M. Variational Methods: applications to nonlinear partial differential equations and Hamiltonian systems, 3rd ed, Springer, Berlin; New York, 2000.

[34] Stubbe, J. (2008) Bound states of two-dimensional Schrödinger-Newton equations. arXiv:0807.4059 [math-ph], arXiv: 0807.4059.

[35] Wen, L. , Tang, X. and Chen, S. Ground state solutions to logarithmic Choquard equations in $\mathbb{R}^{3}$, Math Meth Appl Sci. (2020) mma.6186.

[36] Yu, M., Zhang, M. and Zhang, X. (2018) Fractional Minimization Problem on the Nehari Manifold. Electronic Journal of Differential Equations, 82, 1-21.

[37] Zhang, C. (2019) Trudinger-Moser Inequalities in Fractional Sobolev-Slobodeckij Spaces and Multiplicity of Weak Solutions to the Fractional-Laplacian Equation, Advanced Nonlinear Studies. 19, 197-217. 